\title{
n-Dimensional congruent lattices using necklaces
}

\author{
David Arnas $^{\mathrm{a}, *}$, Daniel Casanova ${ }^{\mathrm{b}}$, Eva Tresaco ${ }^{\mathrm{b}}$ \\ a Massachusetts Institute of Technology, Cambridge, MA 02139, USA \\ ${ }^{\mathrm{b}}$ Centro Universitario de la Defensa - Zaragoza, Crta. Huesca s/n, 50090 Zaragoza, Spain
}

Received 23 January 2020; received in revised form 18 April 2020; accepted 25 April 2020

\begin{abstract}
This work introduces the $n$-dimensional congruent lattices using necklaces, a general methodology to generate uniform distributions in multidimensional modular spaces. The formulation presented in this manuscript constitutes the mathematical foundation of the most used satellite constellation designs, including Walker Constellations, and Lattice and Necklace Flower Constellations. These constellation design models are based on Number Theory and allow to obtain distributions that have some interesting properties of uniformity and large number of symmetries. This work includes the complete formulation of the methodology, proofs for existence and uniqueness of the distribution definitions, and several theorems that focus on the counting possibilities of design for the most common cases of study. (C) 2020 COSPAR. Published by Elsevier Ltd. All rights reserved.
\end{abstract}

Keywords: Satellite constellations; Mathematical models; Number theory; Combinatorics

\section{Introduction}

The generation of distributions of elements in multidimensional spaces while preserving some configuration properties is a complex process due to the large number of variables involved in the problem, which are proportional to the number of elements that are required to be distributed and the number of dimensions considered. This situation is worsened by the difficulty of studying and optimizing distributions that depend on many variables in a reasonable amount of time. An example of this kind of problems is satellite constellation design.

In general, satellite constellation design requires to set the values of six variables per satellite in order to define the state of each spacecraft in the constellation. These variables could be, for example, the positions and velocities of the satellites, or the so called classical elements (semi-major

\footnotetext{
* Corresponding author.

E-mail addresses: arnas@mit.edu (D. Arnas), casanov@unizar.es (D. Casanova), etresaco@unizar.es (E. Tresaco).
}

axis, inclination, eccentricity, right ascension of the ascending node, argument of perigee and mean anomaly). This means that the number of dimensions considered in the problem quickly increases with the number of satellites in the constellation. Moreover, and since satellite constellations are dynamical systems, multitude interrelations appear between satellites during their movement, increasing even more the complexity of these systems.

In the last fifty years, a wide variety of constellation design methodologies have been introduced to simplify the design process and the study of satellite constellations. Examples of that are Walker Constellations (Walker, 1984), Streets of Coverage (Luders, 1961), Draim Constellations (Draim, 1987), Flower Constellations (Mortari et al., 2004; Mortari and Wilkins, 2008; Wilkins and Mortari, 2008), or the kinematically regular satellite networks (Mozhaev, 1973), but there are many others (Ulybyshev, 2008; Lo, 1999; Beste, 1978; Ballard, 1980; Ortore et al., 2017; Dale et al., 2017; Arnas et al., 2017b). The common characteristic of all these design methodologies is that they limit the searching space by imposing a 
set of conditions that are interesting for satellite constellations, such as repeatability of the dynamic, Earth coverage, or the sharing of their space tracks.

Most of the constellation design methodologies rely on performing their satellite distribution using solely real numbers. This seems to be the most natural process to follow since the distribution variables are real numbers (such as distances, angles or velocities). However this is far from true. In a constellation there are an integer number of satellites that are located in an integer number of orbits. Thus, it makes sense to use integers in the definition of the constellation distribution. This is the basic idea behind Lattice Flower Constellations (Avendaño et al., 2013; Avendaño and Mortari, 2012; Davis et al., 2013), to benefit from the properties of Number Theory in order to perform satellite constellation design.

Lattice Flower Constellations have shown its beneficial properties in different problems including telecommunications (Mortari et al., 2011), Earth coverage and global positioning systems (Casanova et al., 2014a). Moreover, the effects of orbital perturbations have also been studied for Lattice Flower Constellations showing that it is easy and feasible to maintain the properties of constellations over time (Casanova et al., 2015; Arnas et al., 2016; Arnas and Casanova, 2020).

However, the number of possibilities that Lattice Flower Constellations can generate is limited by the number of satellites in the constellation. This limitation was overcome by the introduction of the concept of necklace in satellite constellation design (Casanova et al., 2014). The idea was to generate a fictitious constellation with a larger number of satellites than the ones required, and then select a subset of those satellites (the real ones) in such a way that the configuration maintains the properties of uniformity and symmetry from the original Lattice Flower Constellation. Later, and in order to generalize this idea for its application to optimization problems and extend the original applications and design possibilities, Necklace Flower Constellations were introduced (Arnas, 2018; Arnas et al., 2017a,c).

This paper introduces the n-Dimensional congruent lattices using necklaces, a mathematical methodology that allows to generate uniform distributions in a modular space of any dimension, maintaining the properties of uniformity, symmetry and congruence of the resultant configurations. In particular, this methodology represents the generalization of the methodologies introduced in the $2 \mathrm{D}$ (Arnas et al., 2017a), 3D (Arnas et al., 2017c), and 4D (Arnas, 2018) Lattice and Necklace Flower Constellations, making the formulation more compact and robust. In that respect, this manuscript deals also with the problem of existence and uniqueness of the configurations obtained, providing the set of constraints that the parameters of the distribution must fulfill in order to avoid duplicates in the formulation. Additionally, several theorems that focus on the problem of counting the number of possibilities of design under different conditions are presented. As such, they represent the mathematical generalization of the theo- rems introduced for the 2D Necklace Flower Constellations (Arnas et al., 2017a). Furthermore, the theorems included in this work have proven useful to other applications other than satellite constellation design. An example of that is kernel generation for star-trackers under the existence of false stars in their images (Arnas et al., 2017d).

This article is organized as follows. First, a motivation of the manuscript is included in order to link this work to the origins of the problem considered. Second, we introduce the concept of congruent lattices in $n$-dimensional spaces, providing a general formulation to define congruent distributions in multidimensional modular spaces, to find the constraints of the formulation and to derive the number of possibilities of design resultant from this methodology. Then, we present the concept of Necklace and apply it to the congruent lattices generated previously. In that regard, we are interested in finding the distributions that maintain the conditions of congruence from the original lattice configurations. Moreover, we include several theorems for counting the number of possibilities that this formulation allows. Finally, we show the relations between this mathematical methodology with the Lattice and Necklace Flower Constellations.

\section{Motivation of the problem}

As mentioned in the introduction, the work presented in this manuscript is motivated by the difficulties that appear during satellite constellation design when a large number of spacecrafts is considered. Space missions including global coverage, telecommunications, or global positioning systems are examples of applications where this problem becomes important. In particular, these systems require that the resultant distributions are as uniform as possible in order to optimize the number of satellites in orbit.

As a result of that, Walker Constellations have become the most well known satellite constellation design when defining constellations in circular orbits and several orbital planes. The idea behind this distribution is to obtain a configuration of satellites in such a way that no matter which satellite of the constellation is selected, the spacecraft configuration observed from it remains the same. In other words, the relative configuration of the constellation is independent of the satellite that is selected as the reference. This interesting property is achieved by distributing all the satellites using only the right ascension of the ascending node and the mean anomaly as distribution variables, where satellites are located evenly in a given number of equally spaced orbital planes, being the distances between satellites of the same orbit constant. This kind of distribution is in fact, and mathematically speaking, the representation of a congruent lattice (Apostol, 1976; Conway and Sloane, 1999; Birkhoff, 1935) in a two dimensional modular space. Therefore, this work aims to focus on the mathematical aspect of the problem in order to obtain useful information on these distributions and their properties. 
This general idea for the uniform distribution of satellites can be extended to elliptical orbits by introducing the argument of perigee as the third distribution variable. In that case, the objective remains the same, to generate a configuration whose relative distribution is independent on the satellite of the constellation selected. This is the focus of 2D and 3D Lattice Flower Constellations. Additionally, this methodology can be extended by taking into account more distribution variables. Particularly, 4D Lattice Flower Constellations becomes the design tool when the semi-major axes of the orbits are considered or when we want to include the orbit inclination as a distribution parameter.

All these satellite constellation designs generate completely uniform configurations in the chosen distribution variables. However, there are missions where, despite of requiring a mostly uniform distribution, satellites cannot be located evenly in their inertial orbits due to mission requirements. Also, there are many space missions whose mission requirements only allow to define a set of available positions where satellites can be located, needing in addition a uniform distribution of spacecrafts in these available positions to optimize revisiting time, coverage or telecommunication access. For those applications, the notion of necklace (Riordan, 1978; Tucker, 1984; Gilbert and Riordan, 1961) is introduced, using as a base the previous satellite constellation designs. This allows to increase the number of possibilities that these methodologies can provide and allows more freedom during design. For this reason, it seems interesting to study how to extend the notion of necklace into congruent lattices while providing a mathematical foundation to all these satellite constellation design methodologies already in use.

\section{3. n-Dimensional congruent lattice distribution}

The objective of this section is to provide a mathematical foundation for the generation of uniform distributions in modular spaces and extend it to problems where any number of distribution variables is involved. In addition, we are interested in aiming this study to the mathematical generalization of Walker, Draim, Lattice Flower and Necklace Flower Constellations.

Therefore, the first goal of this manuscript is to generate a uniform distribution of points in a n-dimensional space that is subjected to modular arithmetic. This implies three important consequences. First, due to the modular arithmetic affecting the $n$-dimensional space, such space can be regarded as a $(n-1)$-dimensional torus in a $n$ dimensional space. Second, since points cannot be split, there are an integer number of them that are distributed in the space considered. Third, the locations of these points are defined using coordinates in the space, which means that, in order to locate a point, $n$ real numbers are required, being $n$ the number of dimensions of the space. Thus, a relation between a set of integers and the real space must be defined, that is, a lattice.
Let $n$ be the number of dimensions of a space where a congruent lattice is required to be generated. The lattice is defined as an application between $\mathbb{Z}^{n}$ and $\mathbb{R}^{n}$ :

$$
\begin{aligned}
\mathrm{T} 1: \mathbb{Z}^{n} & \longrightarrow \mathbb{R}^{n} \\
\boldsymbol{k} & \mapsto \boldsymbol{V}
\end{aligned}
$$

where $\boldsymbol{k}$ names each point of the distribution, and $\boldsymbol{V}$ locates it in the space considered, that is, it is the set of coordinates of each point of the distribution. Let $i=1, \ldots, n$ be an integer parameter that names each of the dimensions of the space. Then, $\boldsymbol{V}=\left(V_{1}, V_{2}, \ldots, V_{i}, \ldots, V_{n}\right) \in \mathbb{R}^{n}$ is a vector containing the distribution variables, while $\boldsymbol{k}=\left(k_{1}, k_{2}, \ldots, k_{i}, \ldots, k_{n}\right) \in \mathbb{Z}^{n}$ is the vector containing the distribution parameters on each dimension. In particular, $k_{i}$ represents the relative position of the point in the dimension $i \in \mathbb{Z}_{n}$, while $V_{i}$ is the distribution variable in the dimension $i$. Additionally, and without lost of generality, $V_{i}$ is considered to range between $[0,1]$ due to the modular arithmetic of the space. In that regard, it is important to note that spaces with other range sizes can be also obtained by an homotopy of the space defined.

Moreover, and as a mean to define the whole distribution, we denote $\mathcal{K}$ to the number of possible combinations of $\boldsymbol{k}$ for a given lattice configuration that are able to generate different points in the configuration. This implies that the whole lattice distribution can be represented by the following set of points:

$$
\left\{\bigcup_{k}^{\mathcal{K}} \boldsymbol{V}(\boldsymbol{k})\right\}
$$

where $\boldsymbol{V}(\boldsymbol{k})$ are each one of the points defined in the $n$ dimensional space and part of the lattice configuration.

That way, and when related to satellite constellation design, $\boldsymbol{V}$ contains the information about the distribution variables, while $\boldsymbol{k}$ names each satellite of the constellation. For instance, in a 2D Lattice Flower Constellation $2 \pi V_{1}$ represent the relative distribution of the constellation in the right ascension of the ascending node, $2 \pi V_{2}$ is the relative distribution in the mean anomaly, and $k_{1}$ and $k_{2}$ name a satellite of the constellation that is located in the orbital plane $k_{1}$ and the position $k_{2}$ of that orbit. Furthermore, $\mathcal{K}$ represents all the possible combinations of $k_{1}$ and $k_{2}$, that is, it contains the identification of all the satellites from the constellation.

Definition 1. Two lattice configurations are identical if they represent the same points in the space. This means that the set of points defined by:

$$
\left\{\bigcup_{k}^{\mathcal{K}} \boldsymbol{V}(\boldsymbol{k})\right\},
$$

is the same as:

$\left\{\cup_{\boldsymbol{k}^{*}}^{\mathcal{K}^{*}} \boldsymbol{V}^{*}\left(\boldsymbol{k}^{*}\right)\right\}$ 
where $\boldsymbol{V}(\boldsymbol{k})$ and $\boldsymbol{V}^{*}\left(\boldsymbol{k}^{*}\right)$ are the points of two different distributions. This definition is represented as:

$\{\underset{\boldsymbol{k}}{\mathcal{K}} \boldsymbol{V}(\boldsymbol{k})\}=\left\{\bigcup_{\boldsymbol{k}^{*}}^{\mathcal{K}^{*}} \boldsymbol{V}^{*}\left(\boldsymbol{k}^{*}\right)\right\}$.

However, an in order to simplify notation, we refer to two identical configurations as:

$\boldsymbol{V}^{*}\left(\boldsymbol{k}^{*}\right) \equiv \boldsymbol{V}(\boldsymbol{k})$,

for the whole distribution, or:

$V_{i}^{*}\left(\boldsymbol{k}^{*}\right) \equiv V_{i}(\boldsymbol{k})$,

with $i \in\{1, \ldots, n\}$, for each given dimension of the configuration.

Since we are dealing with configurations with a countable number of points distributed, the number of available positions in a given dimension is limited. Therefore, it makes sense to define a parameter to count the number of these available positions. Let $L_{i i}$ be the number of different values of $V_{i}$ provided that $\left\{k_{j} \mid j \neq i\right\}$ are fixed. In other words, $L_{i i}$ represents the number of available positions in the dimension $i$ (or possible values of $V_{i}$ ) under a variation of the distribution parameter $k_{i}$.

Definition 2. A congruent lattice is defined as a lattice whose points fulfill the following condition:

$$
\begin{aligned}
& V_{i}\left(\left\{1, \ldots, k_{m}, \ldots, k_{n}\right\}\right) \\
\equiv & V_{i}\left(\left\{1, \ldots, k_{m}+L_{m m}, \ldots, k_{n}\right\}\right) \quad \forall i, m \in\{1,2, \ldots, n\},
\end{aligned}
$$

which means that a complete rotation in one of the dimensions of the space of configuration generates an identical distribution. In that respect, it is worth noticing that even if two distributions are identical, each point can be defined with a different combination of the values of $\boldsymbol{k}$. In addition, and in order to ease the notation, from now on we will refer to this condition as:

$V_{i}\left(k_{m}\right) \equiv V_{i}\left(k_{m}+L_{m m}\right)$.

Theorem 1. A congruent lattice in a n-dimensional space is defined through an Hermite Normal Form of size $n \times n$, and described as:

$V_{i}=\bmod \left[\frac{1}{L_{i i}}\left(k_{i}-\sum_{j=1}^{i-1}\left(L_{i j} V_{j}\right)\right), 1\right]$,

where $\bmod (a, b)$ is the modulo in base $b$ of $a$, and $L_{i j}$ are the elements of the Hermite Normal Form associated with the distribution.

Proof. A lattice between $\mathbb{Z}^{n}$ and $\mathbb{R}^{n}$ can be defined as an application:

$\mathrm{T} 2$ :

$$
\begin{array}{ccc}
\mathbb{Z}^{n} & \longrightarrow & \mathbb{R}^{n} \\
\left\{\alpha_{1}, \ldots, \alpha_{i}, \ldots, \alpha_{n}\right\} & \mapsto & \left\{V_{1}, \ldots, V_{i}, \ldots, V_{n}\right\}
\end{array}
$$

which, in general, is represented by a system of $n$ independent equations:

$$
\left(\begin{array}{cccc}
P_{1,1} & P_{1,2} & \cdots & P_{1, n} \\
P_{2,1} & P_{2,2} & \cdots & P_{2, n} \\
\vdots & \vdots & \ddots & \vdots \\
P_{n, 1} & P_{n, 2} & \cdots & P_{n, n}
\end{array}\right)\left(\begin{array}{c}
V_{1} \\
V_{2} \\
\vdots \\
V_{n}
\end{array}\right)=\left(\begin{array}{c}
\alpha_{1} \\
\alpha_{2} \\
\vdots \\
\alpha_{n}
\end{array}\right),
$$

where $P_{i, j}$ are a set of integers, and the values of $V_{i}$ can be obtained by an inversion of the matrix (since it is non singular). However, Eq. (12) can produce the same distributions with different combinations of the values of $P_{i, j}$ and $\alpha_{i}$. For this reason, the Hermite Normal Form (Newman, 1972 ) is used in order to avoid possible duplicates in the formulation.

By performing row operations in Eq. (12), the Hermite Normal Form of the system is obtained (Storjohann and Labahn, 1996), that is, a system expressed by a lower triangular matrix applied to the variables of the problem:

$$
\left(\begin{array}{ccccc}
L_{1,1} & 0 & 0 & \cdots & 0 \\
L_{2,1} & L_{2,2} & 0 & \cdots & 0 \\
\vdots & \vdots & \ddots & \ddots & \vdots \\
L_{n-1,1} & L_{n-1,2} & \cdots & L_{n-1, n-1} & 0 \\
L_{n, 1} & L_{n, 2} & \cdots & \cdots & L_{n, n}
\end{array}\right)\left(\begin{array}{c}
V_{1} \\
V_{2} \\
\vdots \\
V_{n-1} \\
V_{n}
\end{array}\right)=\left(\begin{array}{c}
k_{1} \\
k_{2} \\
\vdots \\
k_{n-1} \\
k_{n}
\end{array}\right) .
$$

Note that, due to the process of row operations, $L_{i j}$ and $k_{i}$ are obtained as a linear combination of the former parameters $P_{i j}$ and $\alpha_{i}$ respectively, and thus $L_{i j} \in \mathbb{Z}$ and $k_{i} \in \mathbb{Z}$. Eq. (13) can also be represented in matrix notation as:

$\sum_{j=1}^{i} L_{i j} V_{j}=k_{i}$

and from this equation, an expression for $V_{i}$ can be obtained:

$V_{i}=\frac{1}{L_{i i}}\left(k_{i}-\sum_{j=1}^{i-1} L_{i j} V_{j}\right)$,

which defines a lattice in the space studied. However, Eq. (15) does not fulfill, in general, the conditions for a congruent lattice as it is explained in Definition 2. In particular, using Eq. (15), and applying the condition for congruent lattice:

$V_{i}\left(k_{m}\right) \equiv V_{i}\left(k_{m}+L_{m m}\right)$,

three different cases can be observed:

- If $m>i$ the congruence is fulfilled automatically since $V_{i}$ only depends on terms of $k_{j}$ such that $j \leqslant i$.

- If $m=i$, we have to impose that:

$$
V_{i}\left(k_{i}\right) \equiv V_{i}\left(k_{i}+L_{i i}\right) \quad \forall i \in\{1,2, \ldots, n\},
$$

which leads to: 
$\frac{1}{L_{i i}}\left(k_{i}-\sum_{j=1}^{i-1} L_{i j} V_{j}\right)=\frac{1}{L_{i i}}\left(k_{i}+L_{i i}-\sum_{j=1}^{i-1} L_{i j} V_{j}\right)$,

where both sides of the equation are equal only if $L_{i i}=0$. However, if $L_{i i}=0$, the distribution has no available positions in dimension $i$, and thus, no lattice would be generated. For that reason, an additional property of the space has to be considered to overcome this problem. In particular, if the modular arithmetic of the space is considered and introduced in Eq. (15) the resultant distribution becomes:

$V_{i}=\frac{1}{L_{i i}} \bmod \left(k_{i}-\sum_{j=1}^{i-1} L_{i j} V_{j}, L_{i i}\right)$.

Note that now, this expression is also defining a boundary in the number of different available positions in each dimension. In particular, the condition of congruence lattice is achieved if:

$k_{i}=k_{i}+L_{i i} \bmod \left(L_{i i}\right)$,

which leads to the first boundary of the distribution parameters:

\section{Corollary 1.}

In order to avoid duplicates in the formulation and to obtain a congruent distribution, $k_{i} \in \mathbb{Z}_{L_{i i}}$, where there exist an intrinsic modular arithmetic in the distribution parameters such that:

$k_{i}=k_{i}+L_{i i} \bmod \left(L_{i i}\right)$.

- If $m<i$, the resulting congruent condition leads to:

$\frac{1}{L_{i i}}\left(k_{i}-\sum_{j=1}^{i-1} L_{i j} V_{j}\left(k_{m}\right)\right)=\frac{1}{L_{i i}}\left(k_{i}-\sum_{j=1}^{i-1} L_{i j} V_{j}\left(k_{m}+L_{m m}\right)\right)$,

which is equivalent to the congruence lattice condition since:

$V_{j}\left(k_{m}\right)=V_{j}\left(k_{m}+L_{m m}\right) \quad \forall j, m \in\{1,2, \ldots, n\}$,

as presented in Corollary 1.

Therefore, by using Eq. (19), it is possible to fulfill the congruence condition for all the dimensions of the lattice. Additionally, if the term $L_{i i}$ is introduced inside the modular operator, the following expression is obtained:

$V_{i}=\bmod \left[\frac{1}{L_{i i}}\left(k_{i}-\sum_{j=1}^{i-1} L_{i j} V_{j}\right), 1\right]$.

Note that the definition of the lattice defined by Theorem 1 implies that the point with distribution parameters $k_{i}=L_{i i} \quad \forall i \in \mathbb{Z}_{n}$ is located in the center of the coordinate system of the space, that is, this point represents the origin reference for the whole distribution. Furthermore, it is important to note that by including the modular arithmetic in $L_{i i}$ in the formulation, we have also achieved that the terms $L_{i i}$ represent the number of different positions in $V_{i}$ provided that $\left\{k_{j} \mid j \neq i\right\}$ are fixed. On the other hand, the terms $\left\{L_{i j} \mid i \neq j\right\}$ from the Hermite Normal Form are the configuration numbers, a set of integers that modify the distribution of the lattice, that is, they allow to shift the configuration with respect to different dimensions.

Once the lattice is defined in Theorem 1, a boundary in the parameters is established in order to avoid duplicates in the formulation. In that sense, two sets of boundaries are generated, the first related with the parameter distribution $k_{i}$, and the second related to the possible values of the Hermite Normal Form $L_{i j}$.

Theorem 2. The set of distribution parameters $k_{i}$ is bounded in order to avoid duplicates in the formulation of a congruent lattice. In particular:

$k_{i} \in\left\{1,2, \ldots, L_{i i}\right\}$.

Proof. Let $\boldsymbol{k}$ be a vector containing the distribution parameters where the position $m$ is occupied by $k_{m} \in \mathbb{Z}$, and let $\boldsymbol{k}^{*}$ be the same vector but with the position $m$ occupied by the parameter $k_{m}^{*} \in \mathbb{Z}$ instead of $k_{m}$. Suppose that both distributions are identical in the dimension $m$. Then, using Eq. (24), a relation between both distribution parameters is obtained:

$$
\begin{array}{r}
\bmod \left[\frac{1}{L_{m m}}\left(k_{m}-\sum_{j=1}^{m-1} L_{m j} V_{j}\right), 1\right] \\
=\bmod \left[\frac{1}{L_{m m}}\left(k_{m}^{*}-\sum_{j=1}^{m-1} L_{m j} V_{j}\right), 1\right] .
\end{array}
$$

which can be expanded, leading to:

$\frac{1}{L_{m m}}\left(k_{m}-\sum_{j=1}^{m-1} L_{m j} V_{j}\right)+A=\frac{1}{L_{m m}}\left(k_{m}^{*}-\sum_{j=1}^{m-1} L_{m j} V_{j}\right)$,

where $A$ is an unknown integer. Then, performing some operations in the former expression, the following relation is obtained:

$k_{m}+A L_{m m}=k_{m}^{*}$,

and thus, if $k_{m}$ is defined in the ring of integers $\mathbb{Z}_{\mathbb{L}_{\text {mm }}}=\left\{1,2, \ldots, L_{m m}\right\}, k_{m}^{*}$ can range all $\mathbb{Z}$. On the other hand, the lattices generated in the rest of the dimensions are identical due to the congruence lattice condition (Corollary 1). Consequently, all possible configurations are equivalent to the ones defined by $k_{m} \in\left\{1,2, \ldots, L_{m m}\right\}$.

Theorem 2 also allows to determine the number of different elements that the resultant configuration is able to 
generate. In particular, since $k_{i} \in\left\{1,2, \ldots, L_{i i}\right\}$, the number of element in the distribution is given by the possible combinations of $k_{i}$ with $i \in\{1, \ldots, n\}$, that is:

Corollary 2. The number of elements defined in a congruent configuration where $L_{i i}$ are the number of available positions such that $\left\{k_{j} \mid j \neq i\right\}$ are fixed for each dimension $i \in\{1, \ldots, n\}$ is:

$\prod_{i=1}^{n} L_{i i}$

which corresponds to the number of different combinations of $k_{i}$.

Theorem 3. The set of configuration numbers $L_{i j}$ must be constrained in order to avoid duplicates in the formulation of a congruent lattice. In particular:

$L_{i j} \in\left\{0,1, \ldots, L_{j j}-1\right\} \quad \forall i, j \in \mathbb{Z}_{n}$.

In addition, the relation between two equivalent distributions $L$ and $L^{*}$ is given by:

$L_{i j}^{*}=L_{i j}+\sum_{p=j}^{i-1} A_{p} L_{p j}$

where $A_{p}$ are the integer numbers that are used to perform row operations in the Hermite Normal Form.

Proof. A $n \times n$ integral matrix is in Hermite Normal Form if the matrix is lower triangular (or upper triangular), the elements of the diagonal are positive, and the elements of a column are bounded by $L_{i j} \in\left[0, L_{j j}-1\right]$. If these conditions are met, we can conclude that the matrix is in its Hermite Normal Form and in addition, we can assure that the representation of the system is unique (Newman, 1972). This means that all the equivalent congruent lattice configurations can be generated performing row operations in this Hermite Normal Form (which is unique).

Let $i$ be an arbitrary row in which we want to modify the configuration numbers $L_{i j}$ in such a way that the resultant distribution is identical to the original. In order to obtain the new combination numbers, we perform row operations such that the matrix is still triangular and the diagonal remains unaltered since the diagonal defines the number of elements in each dimension (see Theorem 2). That way, the formulation of the congruent lattice, defined in this work, is maintained no matter the distribution presented.

Let $L$ and $L^{*}$ be two equivalent distributions. Thus, each element of the matrix $L^{*}$ can be expressed by means of the elements of $L$. In particular, the general term of the matrix can be written as:

$L_{i j}^{*}=L_{i j}+\sum_{p=1}^{n} A_{p} L_{p j}$

where $A_{p}$ are the integer numbers that are used to perform the row operations and $n$ is the number of dimensions of the distribution. Since we want the matrix that defines each distribution to be triangular, and with a given set of values in the diagonal, we have to constrain the sum to certain rows. In particular:

$L_{i j}^{*}=L_{i j}+\sum_{p=j}^{i-1} A_{p} L_{p j}$

We can rewrite the former expression into:

$L_{i j}^{*}=L_{i j}+\sum_{p=j+1}^{i-1} A_{p} L_{p j}+A_{j} L_{j j}$

which represents the general expression to obtain all the equivalent distributions of a given configuration. From Eq. (34) and since we can impose the parameters $A_{j}$ to present any integer value, we can transform the expression into:

$L_{i j}^{*}=L_{i j}+\sum_{p=j+1}^{i-1} A_{p} L_{p j} \bmod \left(L_{j j}\right)$,

where it is easy to observe that any distribution can be defined by an equivalent set of parameters that follow this boundary:

$L_{i j}^{*} \in\left\{0,1, \ldots, L_{j j}-1\right\} \quad \forall i, j \in \mathbb{Z}_{n}$,

That way, the boundaries of the configuration numbers $\left(L_{i j} \in\left\{0,1, \ldots, L_{j j}-1\right\}\right)$ are completely defined and also the relations between two equivalent configurations given by this methodology.

\subsection{Number of possible configurations}

Once the lattice and the distribution is completely defined, it is time to evaluate the number of different configurations that can be obtained under the assumption that the number of elements in each dimension $i\left(L_{i i}\right)$ is already known.

Theorem 4. Given a lattice distribution defined by:

$V_{i}=\bmod \left[\frac{1}{L_{i i}}\left(k_{i}-\sum_{j=1}^{i-1}\left(L_{i j} V_{j}\right)\right), 1\right]$,

and for a fixed number of elements distributed in each dimension $i\left(L_{i i}\right)$, the number of different configurations provided by this lattice configuration is:

$\prod_{i=1}^{n} L_{i i}^{(n-i)}$

where $n$ is the number of dimensions of the problem.

Proof. Since the number of elements in each dimension is fixed $L_{i i}$, the only parameters that can modify the distribution are the combination numbers $L_{i j}$ which have the par- 
ticularity that $i \neq j$. Additionally, from Theorem 3, we know that the values of $L_{i j}$ are constrained in order to obtain different configurations. Thus, in the dimension $i$ there are:

$\prod_{j=1}^{i-1} L_{j j} \quad \forall i>1$

possible combinations of the combination numbers. Then, for the complete Hermite Normal Form, we have:

$\prod_{i=2}^{n} \prod_{j=1}^{i-1} L_{j j}$

different configurations of the lattice. The former expression can be rearranged into:

$$
\begin{aligned}
& \left(\prod_{j=1}^{n-1} L_{j j}\right)\left(\prod_{j=1}^{n-2} L_{j j}\right) \cdots\left(\prod_{j=1}^{n-(n-1)} L_{j j}\right) \\
& =L_{11}^{(n-1)} L_{22}^{(n-2)} \cdots L_{(n-1)(n-1)}^{(1)},
\end{aligned}
$$

or in a more compact notation:

$$
\prod_{i=1}^{n} L_{i i}^{(n-i)}
$$

which is the number of different possible configurations in a congruent lattice in a space of $n$ dimensions.

It is important to note that Theorems $1-4$ provide all uniform distributions that can be generated based on a given number of positions in each dimension. This means that it is not possible to generate additional uniform congruent distributions that are not equivalent to the ones provided by this formulation. Moreover, and related to satellite constellation design, this result allows to study all uniform distributions of satellites in a very fast an efficient manner, assuring that all uniform and congruent distributions are assessed.

\section{4. n-Dimensional congruent necklace distributions}

The objective now is to generate a configuration space based on a congruent lattice as described in Eq. (10) and select from it a subset of available positions in such a way that the property of congruence in the distribution is maintained. In order to do that, we first introduce the concept of necklace and some of its basic properties.

Definition 3. A necklace $\mathcal{G}$ is a subset of elements selected from a set of available positions defined under modular arithmetic. It is represented as:

$\mathcal{G} \subseteq \mathbb{Z}_{m}$,

where $m$ is the number of available positions considered.

Definition 4. Two necklaces $\left(\mathcal{G}_{1}\right.$ and $\left.\mathcal{G}_{2}\right)$ are identical (三) if they select the same subset of elements from the available positions:
$\mathcal{G}_{1} \equiv \mathcal{G}_{2} \Longleftrightarrow\left\{\mathcal{G}_{1}\right\}=\left\{\mathcal{G}_{2}\right\} \bmod (m)$

Definition 5. Two necklaces $\left(\mathcal{G}_{1}\right.$ and $\left.\mathcal{G}_{2}\right)$ are equivalent, that is, there is an equivalence relation $\cong$ between them, if:

$\mathcal{G}_{1} \cong \mathcal{G}_{2} \Longleftrightarrow \exists s: \mathcal{G}_{1} \equiv \mathcal{G}_{2}+s$, with $\quad s \in \mathbb{N}_{m}$.

At this point, it is important to remark the differences between identical and equivalent necklaces. A necklace whose elements are reordered but they occupy the same available positions define an identical necklace. Conversely, a necklace that performs a rotation in the available positions generates an equivalent necklace. Additionally, it is easy to derive that two necklaces that are identical, are also equivalent (as seen in Definition 5 if $s \mid m$ ). However, there are other cases where a rotation generates an identical necklace. For that reason, we introduce the concept of symmetry of the necklace.

Definition 6. The symmetry of the necklace is the minimum number of positions that a given necklace has to rotate in order to obtain an identical necklace. The symmetry of a given necklace $\mathcal{G}$ is represented by $\operatorname{Sym}(\mathcal{G})$ and its mathematical expression is provided by the following equation:

$\operatorname{Sym}(\mathcal{G})=\min \{1 \leqslant r \leqslant m: \mathcal{G}+r \equiv \mathcal{G}\}$.

This definition implies that given a necklace $\mathcal{G}$ and applying a rotation of $r: \operatorname{Sym}(\mathcal{G}) \mid r$ (that is, $\operatorname{Sym}(\mathcal{G})$ divides $r$ ) generates an identical necklace (see Definition 4) which is also equivalent to $\mathcal{G}$ (see Definition 5). In addition, this means that the maximum number of rotations that we can apply to the necklace $\mathcal{G}$ without generating an identical necklace is $\operatorname{Sym}(\mathcal{G})-1)$. This constraint allows to define the shifting parameter, a distribution parameter whose objective is the definition of the different rotations that a given necklace can perform in its available positions while avoiding a duplicity in the formulation.

Definition 7. The shifting parameter $S$ is the minimum representation of any rotation that a necklace can perform in its available positions. As a consequence of the definition of symmetry of the necklace and the modular arithmetic of the problem, the shifting parameter is constrained such that:

$S \in\{0,1, \ldots,(\operatorname{Sym}(\mathcal{G})-1)\}$.

In order to clarify the previous mathematical concepts related to necklaces, a simple example is presented in here. Imagine that we have a table with four chairs around it, and at some point two people try to take a seat. If we number the chairs, the combinations in which chairs can be occupied are: $\{1,2\},\{1,3\},\{1,4\},\{2,3\},\{2,4\}, \quad$ and $\{3,4\}$. These are all the combinations of necklaces that we can generate from this problem. If once they have taken a seat we allow them to exchange their seat with their companion, we obtain an identical necklace, that is, for instance $\{1,2\} \equiv\{2,1\}$. However, while sitting in a table, most people are more interested in knowing their relative 
position with respect to other people. If that is the case, there exist only two possible combinations. In the first one, both persons are close to each other. This is represented by solutions $\{1,2\},\{1,4\},\{2,3\}$, and $\{3,4\}$ which are equivalent necklaces. Alternatively, they can take a seat in front of each other, which is represented by combinations $\{1,3\}$ and $\{2,4\}$, being these necklaces also equivalent between them. Finally, and regarding the symmetry of these necklaces, the solution where these people is seated one in front of the other has a symmetry of 2 , that is, if we walk around the table a distance equivalent to two consecutive chairs, we will observe an equivalent configuration. On the other hand, when people is seated close to each other, we need to do a complete turn around to observe an equivalent distribution, that is, the symmetry of these necklaces is 4. A graphical representation of this can be seen in Fig. 1 where the white circles represent empty chairs and the black ones are the chairs occupied by people.

If we relate this concept to satellite constellation design, the available positions (chairs) are the combination of distribution variables (for instance, mean anomaly and right ascension of the ascending node) where a satellite can be located in order to fulfill a given mission, or a set of defined slots from a control strategy for the constellation. On the other hand, the necklace represents the subset of available positions in which we locate a satellite (the persons from the previous example). In here, it is important to note that necklaces can be applied to any distribution variable (or dimension), for instance the mean anomaly (positions in a given orbit), the right ascension of the ascending node (orbital planes in use), or the argument of perigee (different orientations of the orbits that are in the same orbital plane). This allows to define both uniform and nonuniform distributions in terms of combinations of necklaces.

With the definitions and properties of the necklace already defined, it is now possible to find all the different movements that each necklace introduced in the lattice is able to perform in order to maintain the property of congruence in the lattice. As before, we are very interested in avoiding duplicates in the formulation both for counting purposes as for efficiency in the study.

Let $\mathcal{G}_{i}$ be a necklace defined in the dimension $i$ of the space, where $\mathcal{G}_{i}$ is represented as a vector of dimension

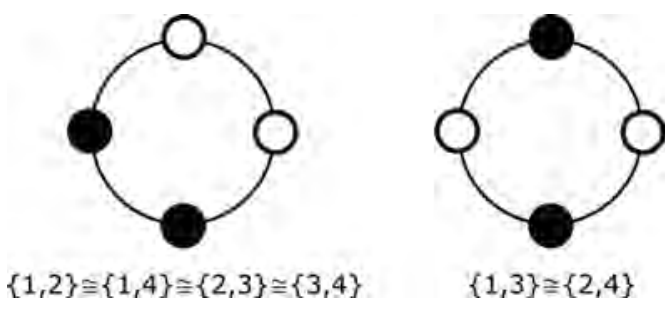

Fig. 1. Example of necklace.
$N_{i}$ which contains the information of the positions occupied from the available positions. Note that $N_{i}$ also corresponds to the number of different elements in the dimension $i$ provided that $\left\{k_{j} \mid j \neq i\right\}$ are fixed. That is, the necklace $\mathcal{G}_{i}$ is a subset from the set of available locations in $\mathbb{Z}_{L_{i i}}$ :

$\mathcal{G}_{i} \subseteq\left\{1, \ldots, L_{i i}\right\}$,

where $\left|\mathcal{G}_{i}\right|=N_{i}$ is the number of elements in the necklace $\mathcal{G}_{i}$. In order to simplify notation, we refer to the elements of the necklace as:

$\mathcal{G}_{i}=\left\{\mathcal{G}_{i}(1), \ldots, \mathcal{G}_{M}\left(k_{i}^{*}\right), \ldots, \mathcal{G}_{i}\left(N_{i}\right)\right\}$,

where without lost of generality are ordered in such a way that:

$1 \leqslant \mathcal{G}_{i}(1) \leqslant \ldots \leqslant \mathcal{G}_{i}\left(k_{i}^{*}\right) \leqslant \ldots \leqslant \mathcal{G}_{i}\left(N_{i}\right) \leqslant L_{i i}$,

being the index $k_{i}^{*} \in\left\{1, \ldots, N_{i}\right\}$ an integer modulo $N_{i}$ (that is, $k_{i}^{*}+N_{i}$ is the same index as $k_{i}^{*}$ ) that names each element inside the necklace. Therefore, it is possible to compute the number of elements in the distribution as the number of different combinations that $k_{i}^{*}$ can provide, that is:

Corollary 3. The number of elements defined in a congruent configuration using necklaces is:

$\prod_{i=1}^{n} N_{i}$

where $N_{i}$ is the number of occupied positions in each dimension $i \in\{1, \ldots, n\}$ such that $\left\{k_{j}^{*} \mid j \neq i\right\}$ are fixed.

This allows the unequivocal definition of a map $\left(\mathcal{G}_{i}\right)$ that describes where the elements of the necklace are located from a set of available positions:

$$
\begin{array}{ccc}
\mathcal{G}_{i}: & \mathbb{Z}_{N_{i}} \longrightarrow & \mathbb{Z}_{L_{i i}} \\
k_{i}^{*} \mapsto & \mathcal{G}_{i}\left(k_{i}^{*}\right) .
\end{array}
$$

Therefore, we use $\mathcal{G}_{i}\left(k_{i}^{*}\right)$ to relate a position in a necklace with its correspondent location in the available positions. Additionally, since the modular arithmetic is affecting the necklace:

$\mathcal{G}_{i}\left(k_{i}^{*}\right)=\mathcal{G}_{i}\left(\bmod \left(k_{i}^{*}+N_{i}, N_{i}\right)\right)$.

which represents a complete rotation in the dimension $i$. Note that such movement of the necklace is equivalent to a rotation in the available positions as defined by Corollary 1 :

$k_{i}=k_{i}+L_{i i} \bmod \left(L_{i i}\right)$,

due to the fact that both formulations represent the same action. In addition, by applying the definition of symmetry of the necklace (Definition 6), the former equation can be defined in terms of the symmetry of the necklace in the dimension $i\left(\operatorname{Sym}\left(\mathcal{G}_{i}\right)\right)$ and the necklace $\mathcal{G}_{i}$ : 
$\mathcal{G}_{i} \equiv \mathcal{G}_{i}+\operatorname{ASym}\left(\mathcal{G}_{i}\right)$

where $A$ is an unknown integer. Thus, the following corollary can be derived:

Corollary 4. The modular arithmetic governing the rotations in the set of available positions subjected to a given necklace is:

$\mathcal{G}_{i} \equiv \mathcal{G}_{i}+\operatorname{Sym}\left(\mathcal{G}_{i}\right)$

Note that since a complete rotation over the available positions generates an identical distribution, $L_{i i}$ must be a multiple of $\operatorname{Sym}(\mathcal{G})$ due to the fact that $\operatorname{Sym}(\mathcal{G})$ is the minimum rotation to generate an identical necklace. This implies that $\operatorname{Sym}(\mathcal{G}) \mid L_{i i}$.

On the other hand, we define a set of movements for each necklace in its dimension in order to provide a methodology in the formulation to shift the necklaces over the available positions with respect to the movement performed in other dimensions. Let the shifting matrix $S$ be a lower triangular matrix of size $n \times n$ composed entirely of integer elements, where the term $S_{i j} \in \mathbb{Z}$ is the shifting parameter (see Definition 7) that relates the movement of the necklace $\mathcal{G}_{i}$ with the movement performed in the dimension $j$. This means that the movement of the necklace $\mathcal{G}_{i}$ is only affected by the movement of necklaces $\mathcal{G}_{j}$ located in dimensions where $j<i$ (as was previously the case with the variables $V_{i}$ and the distribution parameters $k_{i}$ ).

Theorem 5. A congruent lattice in a n-dimensional modular space based on a necklace formulation is defined by the following expression:

$$
\begin{aligned}
\sum_{j=1}^{i} L_{i j} V_{j} & =k_{i} ; \\
k_{i} & =\bmod \left[\mathcal{G}_{i}\left(k_{i}^{*}\right)+\bmod \left(\sum_{j=1}^{i-1} S_{i j} k_{j}, \operatorname{Sym}\left(\mathcal{G}_{i}\right)\right), L_{i i}\right],
\end{aligned}
$$

where $\mathcal{G}_{i}$ is the necklace in the dimension $i, k_{i}^{*}$ is the distribution parameter inside the necklace, and $S_{i j}$ is the shifting parameter of the necklace $\mathcal{G}_{i}$ with respect to the dimension $j$.

Proof. An application between the available locations $k_{i}$ and the necklace elements $\mathcal{G}_{i}\left(k_{i}^{*}\right)$ can be defined as done in Eq. (52):

$$
\begin{array}{cc}
\text { T3: } & \left(\mathbb{Z}_{N_{1}} \times \mathbb{Z}_{N_{2}} \times \ldots \times \mathbb{Z}_{N_{n}}\right) \rightarrow\left(\mathbb{Z}_{L_{11}} \times \mathbb{Z}_{L_{22}} \times \ldots \times \mathbb{Z}_{L_{n n}}\right) \\
\left(k_{1}^{*}, k_{2}^{*}, \ldots, k_{n}^{*}\right) \mapsto \quad\left(k_{1}, k_{2}, \ldots, k_{n}\right),
\end{array}
$$

where the relation between the distribution parameters in the available positions $k_{i}$, and the elements of the necklace is described as:
$k_{i}=\mathcal{G}_{i}\left(k_{i}^{*}\right)+\sum_{j=1}^{i-1} S_{i j} k_{j}$

being $\mathcal{G}_{i}\left(k_{i}^{*}\right)$ the term that provides the information about the structure of the necklace in the dimension, while the term $\sum_{j=1}^{i-1} S_{i j} k_{j}$ represents all the possible dependencies between the necklace $\mathcal{G}_{i}$ with the movement of the configuration in other dimensions. Note that the summation in Eq. (59) is not defined when $i \leqslant j$ since $S_{i j}=0$ for those cases and thus, these operations in the summation are not performed. In addition, and due to the existence of symmetries in the necklace, there are movements that generate identical configurations. Thus, using Corollary 1 and Corollary 4:

$k_{i}=\bmod \left[\mathcal{G}_{i}\left(k_{i}^{*}\right)+\bmod \left(\sum_{j=1}^{i-1} S_{i j} k_{j}, \operatorname{Sym}\left(\mathcal{G}_{i}\right)\right), L_{i i}\right]$,

which avoids the duplicates of configurations for different sets of shifting parameters.

Now, we introduce Eq. (60) into the initial lattice defined in Eq. (14), obtaining:

$$
\begin{aligned}
\sum_{j=1}^{i} L_{i j} V_{j} & =k_{i} \\
k_{i} & =\bmod \left[\mathcal{G}_{i}\left(k_{i}^{*}\right)+\bmod \left(\sum_{j=1}^{i-1} S_{i j} k_{j}, \operatorname{Sym}\left(\mathcal{G}_{i}\right)\right), L_{i i}\right] .
\end{aligned}
$$

Note that the former expression is a recursive function in the different dimensions of the space in study due to the fact that both $L_{i j}$ and $S_{i j}$ are zero when $j>i$.

Theorem 6. The shifting parameters that allow the lattice defined by:

$$
\begin{aligned}
\sum_{j=1}^{i} L_{i j} V_{j} & =k_{i} ; \\
k_{i} & =\bmod \left[\mathcal{G}_{i}\left(k_{i}^{*}\right)+\bmod \left(\sum_{j=1}^{i-1} S_{i j} k_{j}, \operatorname{Sym}\left(\mathcal{G}_{i}\right)\right), L_{i i}\right],
\end{aligned}
$$

to be congruent must fulfill the following relation:

$\operatorname{Sym}\left(\mathcal{G}_{i}\right) \mid S_{i j} L_{j j}-\left(L_{i j}-\sum_{q=j+1}^{i-1} S_{i q} L_{q j}\right)$.

Proof. In order to derive the theorem, we focus on a given dimension $i$ where the congruence condition seen in Definition 2 is applied:

$V_{i}\left(k_{j}\right) \equiv V_{i}\left(k_{j}+L_{j j}\right) \quad \forall i, j \in\{1,2, \ldots, n\}$. 
This represents a complete rotation of the configuration in the dimension $j$ while the other dimensions remain unrotated. This means also that in addition to the congruence condition this relation is also true:

$V_{i}\left(k_{j}\right)=V_{i}\left(k_{j}+L_{j j}\right) \quad \forall i \neq j$,

since only the dimension $i$ is subjected to a movement.

From the lattice defined in Eq. (57) three different cases can be observed: the case in which $j>i$, the case $j=i$ and the case $j<i$. For all the cases of study we consider an original lattice before the complete rotation in the dimension $j$ as:

$$
\begin{aligned}
\sum_{p=1}^{i} L_{i p} V_{p}\left(k_{j}\right) & =k_{i}\left(k_{j}\right) \\
k_{i}\left(k_{j}\right) & =\mathcal{G}_{i}\left(k_{i}^{*}\right)+\sum_{p=1}^{i-1} S_{i p} k_{p}\left(k_{j}\right)+A_{i} \operatorname{Sym}\left(\mathcal{G}_{i}\right),
\end{aligned}
$$

where $V_{p}\left(k_{j}\right)$ and $k_{p}\left(k_{j}\right)$ represent the variable and the distribution parameters of the original configuration, and $A_{i}$ is an unknown integer. On the other hand, the lattice after the complete rotation in the dimension $j$ is:

$$
\begin{aligned}
\sum_{p=1}^{i} L_{i p} V_{p}\left(k_{j}+L_{j j}\right) & =k_{i}\left(k_{j}+L_{j j}\right) \\
k_{i}\left(k_{j}+L_{j j}\right) & =\mathcal{G}_{i}\left(k_{i}^{*}\right)+\sum_{p=1}^{i-1} S_{i p} k_{p}\left(k_{j}+L_{j j}\right)+B_{i} \operatorname{Sym}\left(\mathcal{G}_{i}\right),
\end{aligned}
$$

where $V_{p}\left(k_{j}+L_{j j}\right)$ represents the variable in the dimension $p$ affected by a complete rotation performed in the dimension $j$, and $k_{p}\left(k_{j}+L_{j j}\right)$ is the modified distribution parameter $k_{p}$ after the rotation. Finally $B_{i}$ is an unknown integer generated as a consequence of the modular arithmetic of the problem.

- If $j>i$, the difference between both lattices is:

$\sum_{p=1}^{i} L_{i p} V_{p}\left(k_{j}+L_{j j}\right)-\sum_{p=1}^{i} L_{i p} V_{p}\left(k_{j}\right)=\sum_{p=1}^{i} L_{i p}\left[V_{p}\left(k_{j}+L_{j j}\right)-V_{p}\left(k_{j}\right)\right]=0$,

which implies that the rotation in the dimension $j$ does not affect $k_{i}$. That is:

$k_{p}\left(k_{j}+L_{j j}\right)=k_{p}\left(k_{j}\right) \quad \forall p<j$.

Regarding the second expression of the lattice, we compare the original and the rotated configuration, obtaining:

$0=\sum_{p=1}^{i-1} S_{i p}\left[k_{p}\left(k_{j}+L_{j j}\right)-k_{p}\left(k_{j}\right)\right]+\left(B_{i}-A_{i}\right) \operatorname{Sym}\left(\mathcal{G}_{i}\right)$,

which means that no constraint has to be imposed in the shifting parameters for the fulfillment of the congruent condition.
- If $j=i$, the difference between both lattices is:

$\sum_{p=1}^{j} L_{j p}\left[V_{p}\left(k_{j}+L_{j j}\right)-V_{p}\left(k_{j}\right)\right]=L_{j j}$,

since $L_{j j}=k_{j}\left(k_{j}+L_{j j}\right)-k_{j}\left(k_{j}\right)$ (a complete rotation in this dimension). In addition, the former equation can be rearranged into:

$L_{j j}\left[V_{j}\left(k_{j}+L_{j j}\right)-V_{j}\left(k_{j}\right)\right]+\sum_{p=1}^{j-1} L_{j p}\left[V_{p}\left(k_{j}+L_{j j}\right)-V_{p}\left(k_{j}\right)\right]=L_{j j}$,

and using Eq. (65), we obtain:

$V_{j}\left(k_{j}+L_{j j}\right)-V_{j}\left(k_{j}\right)=1$

that represents the complete rotation in the dimension $i$. It is clear that since the variables $V_{i}$ are subjected to a modulo 1 arithmetic, $V_{i}\left(k_{i}+L_{i i}\right) \cong V_{i}\left(k_{i}\right)$. However, this result affects other dimensions as it is shown later. On the other hand, from the differences between the second terms of the lattice of the original and rotated configurations:

$$
\begin{aligned}
L_{j j}= & \sum_{p=1}^{j-1} S_{j p}\left[k_{p}\left(k_{j}+L_{j j}\right)-k_{p}\left(k_{j}\right)\right]+\left(B_{j}\right. \\
& \left.-A_{j}\right) \operatorname{Sym}\left(\mathcal{G}_{j}\right)
\end{aligned}
$$

where applying the condition presented in Eq. (69) we derive that:

$L_{j j}=\left(B_{j}-A_{j}\right) \operatorname{Sym}\left(\mathcal{G}_{j}\right)$,

and thus, no additional constraint must be imposed since $\operatorname{Sym}\left(\mathcal{G}_{j}\right) \mid L_{j j}$.

- If $j<i$, the difference between both lattices is:

$$
\begin{aligned}
& \sum_{q=1}^{i} L_{i q} V_{q}\left(k_{j}+L_{j j}\right)-\sum_{q=1}^{i} L_{i q} V_{q}\left(k_{j}\right) \\
& =k_{i}\left(k_{j}+L_{j j}\right)-k_{i}\left(k_{j}\right),
\end{aligned}
$$

which is equal to:

$$
\sum_{q=1}^{i} L_{i q}\left[V_{q}\left(k_{j}+L_{j j}\right)-V_{q}\left(k_{j}\right)\right]=k_{i}\left(k_{j}+L_{j j}\right)-k_{i}\left(k_{j}\right),
$$

and if rearranged leads to:

$$
\begin{aligned}
& \sum_{q=1}^{j-1} L_{i q}\left[V_{q}\left(k_{j}+L_{j j}\right)-V_{q}\left(k_{j}\right)\right]+L_{i j}\left[V_{j}\left(k_{j}+L_{j j}\right)-V_{j}\left(k_{j}\right)\right] \\
& +\sum_{q=j+1}^{i} L_{i q}\left[V_{q}\left(k_{j}+L_{j j}\right)-V_{q}\left(k_{j}\right)\right]=k_{i}\left(k_{j}+L_{j j}\right)-k_{i}\left(k_{j}\right),
\end{aligned}
$$

which can be simplified using Eqs. (65) and (73), to obtain:

$L_{i j}=k_{i}\left(k_{j}+L_{j j}\right)-k_{i}\left(k_{j}\right)$. 
On the other hand, from the differences in the second term of the lattice of both configurations:

$$
\begin{aligned}
k_{i}\left(k_{j}+L_{j j}\right)-k_{i}\left(k_{j}\right)= & \sum_{p=1}^{i-1} S_{i p}\left[k_{p}\left(k_{j}+L_{j j}\right)-k_{p}\left(k_{j}\right)\right] \\
& +\left(B_{j}-A_{j}\right) \operatorname{Sym}\left(\mathcal{G}_{j}\right),
\end{aligned}
$$

where using Eq. (79) and (69) leads to:

$$
\begin{aligned}
L_{i j}= & \sum_{p=j}^{i-1} S_{i p}\left[k_{p}\left(k_{j}+L_{j j}\right)-k_{p}\left(k_{j}\right)\right]+\left(B_{j}\right. \\
& \left.-A_{j}\right) \operatorname{Sym}\left(\mathcal{G}_{j}\right) .
\end{aligned}
$$

In addition, the former expression can be rearranged into:

$$
\begin{aligned}
L_{i j} & =S_{i j}\left[k_{j}\left(k_{j}+L_{j j}\right)-k_{j}\left(k_{j}\right)\right] \\
& +\sum_{p=j+1}^{i-1} S_{i p}\left[k_{p}\left(k_{j}+L_{j j}\right)-k_{p}\left(k_{j}\right)\right]+\left(B_{j}-A_{j}\right) \operatorname{Sym}\left(\mathcal{G}_{j}\right),
\end{aligned}
$$

where:

$$
k_{j}\left(k_{j}+L_{j j}\right)-k_{j}\left(k_{j}\right)=L_{j j},
$$

representing the complete rotation in the dimension $j$, while:

$$
k_{p}\left(k_{j}+L_{j j}\right)-k_{p}\left(k_{j}\right)=L_{p j}
$$

as seen in Eq. (79). Thus, Eq. (82) can be written as:

$$
L_{i j}=S_{i j} L_{j j}+\sum_{p=j+1}^{i-1} S_{i p} L_{p j}+\left(B_{j}-A_{j}\right) \operatorname{Sym}\left(\mathcal{G}_{j}\right) \text {, }
$$

or, in a more compact manner:

$$
\operatorname{Sym}\left(\mathcal{G}_{i}\right) \mid S_{i j} L_{j j}-\left(L_{i j}-\sum_{q=j+1}^{i-1} S_{i q} L_{q j}\right),
$$

which is an expression that relates the values of the shifting parameters with respect to elements of the Hermite Normal Form and other shifting parameters. Note that the expression is a recursive function.

Thus, and as seen from the three cases studied, the only constraint that affects the shifting parameters is:

$$
\operatorname{Sym}\left(\mathcal{G}_{i}\right) \mid S_{i j} L_{j j}-\left(L_{i j}-\sum_{q=j+1}^{i-1} S_{i q} L_{q j}\right) .
$$

\subsection{Number of possible configurations}

This section focuses on the problem of counting the number of different configurations that the $n$-dimensional congruent lattices using necklaces can generate. In that regard, three general scenarios are considered, which represents the most common cases of study:
- Fixing the set of necklaces $\mathcal{G}_{i}$ for all $i \in\{1, \ldots, n\}$, and the Hermite Normal Form.

- Fixing the set of necklaces $\mathcal{G}_{i}$ and $L_{i i}$ for all $i \in\{1, \ldots, n\}$.

- Fixing the sizes of the necklaces $N_{i}=\left|\mathcal{G}_{i}\right|$ and $L_{i i}$ for all $i \in\{1, \ldots, n\}$.

However, before starting with this study, we are previously required to determine the number of necklace configurations, of a given size, that can be generated in a given dimension. This result will be used in the scenarios proposed. Let $L$ be a set of available positions in a given dimension, and let $\mathcal{G}$ be a necklace comprised of $N$ elements selected from these locations. This means that $\mathcal{G} \subseteq\{1, \ldots, L\}$ where $|\mathcal{G}|=N$.

Theorem 7. The number of different necklaces under the equivalence relation $\cong$ and which are comprised by $N$ elements selected from $L$ available positions is:

$$
\sum_{\substack{g\left|L \\
\frac{L}{g}\right| N}}^{L} \frac{1}{g}\left[\left(\begin{array}{c}
g \\
\frac{N}{L} g
\end{array}\right)-\sum_{\substack{g^{\prime}=1 \\
g^{\prime}\left|g \\
\frac{L}{g^{\prime}}\right| N}}^{g-1} \frac{g^{\prime}}{L}\left|\operatorname{Fix}\left(g^{\prime}\right)\right|\right],
$$

where $g=\operatorname{Sym}(\mathcal{G})$ are the possible symmetries in the problem considered and $|\mathrm{Fix}(s)|$ is the number of elements contained in the Fix of a given symmetry s, and which is provided by:

$$
|\operatorname{Fix}(s)|=\frac{L}{s}\left[\left(\begin{array}{c}
s \\
\frac{N}{L} s
\end{array}\right)-\sum_{\substack{s^{\prime}=1 \\
s^{\prime}\left|s \\
\frac{L}{s^{\prime}}\right| N}}^{s-1} \frac{s^{\prime}}{L}\left|\operatorname{Fix}\left(s^{\prime}\right)\right|\right] .
$$

Proof. Let $+\mathbb{Z}_{L}$ be the possible different actions that can be applied to a given necklace. In the problem considered, these actions represent the different rotations that a defined necklace $\mathcal{G}$ can perform over the set of available positions $\mathbb{Z}_{L}$. In addition, let $G=\mathbb{Z}_{L}$ be the group containing the actions that can be applied to any necklace defined in $L$ available positions. Therefore, a map $\phi$ can be described as:

$$
\begin{array}{ccc}
\phi: & G \times X \rightarrow & X \\
(g, x) \mapsto & x+g \bmod (L) .
\end{array}
$$

Once this map is defined, we aim to apply Burnside's Lemma (Tucker, 1984) to this application. In order to do that, we first require to determine the $|\operatorname{Fix}(g)|$.

The Fix of an action is defined as the set of elements that remain unchanged under the application of that action. 
Therefore, from the definition of symmetry of a necklace (see Eq. (46)), it can be derived that the only values of $g \in G$ that have elements in the $\operatorname{Fix}(X)$ are those that have symmetries, in other words, these elements must fulfill the condition $g=\operatorname{Sym}(\mathcal{G})$. This implies that the only elements that contribute to the Fix are those where $g \mid L$ and $\frac{L}{g} \mid N$.

First, we deal with the problem of finding the number of different necklaces that are associated with a given symmetry of the necklace. To that end, we focus on a particular value of $g$ and compute its Fix. Since $g=\operatorname{Sym}(\mathcal{G})$ is an integer number that divides $L$, the problem can be also regarded as the repetition of a pattern of size $g$ that is repeated $L / g$ times. Therefore, and since the pattern is repeated, there must be $\mathrm{Ng} / L$ elements from the necklace in each pattern. Then, the number of pattern combinations of size $g$, denoted by $P C(g)$, is:

$P C(g)=\left(\begin{array}{c}g \\ \frac{N}{L} g\end{array}\right)$.

Additionally, since the pattern is repeated inside the necklace (due to its symmetry), each pattern is able to rotate $L / g$ times and still generate the same configuration. Therefore, there are:

$\frac{L}{g} P C(g)=\frac{L}{g}\left(\begin{array}{c}g \\ \frac{N}{L} g\end{array}\right)$,

different combinations of $N$ elements with symmetry $g$ selected from $L$ available positions. However, this quantity is also including some elements that can be generated under other symmetries. In that respect, it is important to remember that the symmetry of the necklace was defined as the minimum rotation in order to obtain an identical necklace (see Definition 6). Therefore, the elements related with these other symmetries must be removed in order to avoid duplicates during the process. As an example of that, let $L=6$ and $N=3$. If we select $g=6$ as the symmetry in study, the number of combinations obtained in Eq. (91) also includes the combination of elements that has symmetry of $g=3$, that is, necklaces $\{1,4\},\{2,5\}$ and $\{3,6\}$. For that reason, we have to take into account this effect. In that regard, we define the symmetry of the necklace as the smallest symmetry that a necklace has.

Moreover, the Fix represents the number of combinations of $N$ elements with a particular symmetry $g$, since these elements are invariant under a rotation $g$. In addition, necklaces with symmetry $g$ are generated based on patterns of size $g$ (see Eq. (91)). Thus, under a symmetry $g$ there must be:

$P C(g)=\frac{g}{L}|\operatorname{Fix}(g)|$,

different patterns. As commented before, from the number of patterns associated with a given symmetry, it is necessary to remove, during the counting process, those elements that have other symmetries such that $g^{\prime}<g$, thus:

$$
P C(g)=\left(\begin{array}{c}
g \\
\frac{N}{L} g
\end{array}\right)-\sum_{\substack{g^{\prime}=1 \\
g^{\prime}\left|g \\
\frac{L}{g^{\prime}}\right| N}}^{g-1} \frac{g^{\prime}}{L}\left|\operatorname{Fix}\left(g^{\prime}\right)\right|,
$$

where the summation is done over the symmetries $g^{\prime}$ such that $g^{\prime} \mid g$ and $\frac{L}{g} \mid N$. Note that these constraints are imposed to $g^{\prime}$ due to the fact that the conditions of symmetry are still applied inside the summation.

That way, by combining the results from Eqs. (93) and (94) the $|\operatorname{Fix}(g)|$ is obtained:

$$
|\operatorname{Fix}(g)|=\frac{L}{g}\left[\left(\begin{array}{c}
g \\
\frac{N}{L} g
\end{array}\right)-\sum_{\substack{g^{\prime}=1 \\
g^{\prime}\left|g \\
\frac{L}{g^{\prime}}\right| N}}^{g-1} \frac{g^{\prime}}{L}\left|\operatorname{Fix}\left(g^{\prime}\right)\right|\right] .
$$

Eq. (95) is a recursive function that provides the number of elements contained in the Fix under the action $g$. This result allows to determine the number of different necklaces under a symmetry $g$ by the use of Burnside's Lemma. Since $G=\mathbb{Z}_{L}$, the number of different necklaces is:

$$
\begin{gathered}
\frac{1}{L} \sum_{g=1}^{L}|\operatorname{Fix}(g)|, \\
g \mid L \\
\quad \frac{L}{g} \mid N
\end{gathered}
$$

which can be rewritten using Eq. (95) to obtain:

$$
\sum_{\substack{g=1 \\
\frac{L}{g} \mid N}}^{L} \frac{1}{g}\left[\left(\begin{array}{c}
g \\
\frac{N}{L} g
\end{array}\right)-\sum_{\substack{g^{\prime}=1 \\
g^{\prime}\left|g \\
\frac{L}{g^{\prime}}\right| N}}^{g-1} \frac{g^{\prime}}{L}\left|\operatorname{Fix}\left(g^{\prime}\right)\right|\right] .
$$

Once Theorem 7 is presented, it is now possible to introduce the counting theorems for congruent lattices using necklaces. Each condition studied and theorem is presented in a different subsection.

\subsubsection{Fixing $\mathcal{G}_{i}$ for all $i \in\{1, \ldots, n\}$ and the Hermite Normal Form}

In this section we study the problem of counting the number of different congruent configurations that can be generated under the condition that the available positions in the space are fixed, and the patterns that are applied in each dimension are already defined. This means that the necklaces $\mathcal{G}_{i}$ and the complete Hermite Normal Form are fixed for the whole configuration. This problem is 
equivalent to computing the number of different shifting parameters $S_{i j}$ that are compatible with Eq. (63).

Theorem 8. Given a set of necklaces $\mathcal{G}_{i}$ in each dimension of the space such that $i \in\{1, \ldots, n\}$, and a fixed Hermite Normal Form, there exist congruent configurations in the available distributions if and only if:

$$
\begin{aligned}
& \operatorname{gcd}\left(\operatorname{Sym}\left(\mathcal{G}_{i}\right), L_{j j}\right) \mid\left(L_{i j}-\sum_{q=j+1}^{i-1} S_{i j} L_{q j}\right), \forall\left\{i, j \in \mathbb{Z}_{n}: i\right. \\
& >j\} .
\end{aligned}
$$

Furthermore, if that condition is fulfilled, the number of different configurations is provided by:

$\prod_{i=2}^{n} \prod_{j=1}^{i-1} \operatorname{gcd}\left(\operatorname{Sym}\left(\mathcal{G}_{i}\right), L_{j j}\right)$

Proof. Eq. (63) can be expressed as:

$\operatorname{ASym}\left(\mathcal{G}_{i}\right)+L_{j j} S_{i j}=L_{i j}-\sum_{q=j+1}^{i-1} S_{i j} L_{q j}$,

where $A$ is an unknown integer. As it can be seen, Eq. (100) can be regarded as a linear Diophantine equation where $A$ and $S_{i j}$ are the variables of study and the rest of parameters are already known. Thus, using Bézout's identity, it can be concluded that a solution to the equation exists if and only if:

$\operatorname{gcd}\left(\operatorname{Sym}\left(\mathcal{G}_{i}\right), L_{j j}\right) \mid L_{i j}-\sum_{q=j+1}^{i-1} S_{i j} L_{q j}$.

In the case the former expression is fulfilled, all the possible solutions of Eq. (100) can be expressed as:

$$
\begin{aligned}
\left(S_{i j}\right)_{\lambda} & =\left(S_{i j}\right)_{0}+\lambda \Delta l, \quad \text { with } \quad \Delta l=\frac{\operatorname{Sym}\left(\mathcal{G}_{i}\right)}{\operatorname{gcd}\left(\operatorname{Sym}\left(\mathcal{G}_{i}\right), L_{j j}\right)}, \\
(A)_{\lambda} & =(A)_{0}-\lambda \frac{L_{j j}}{\operatorname{gcd}\left(\operatorname{Sym}\left(\mathcal{G}_{i}\right), L_{j j}\right)},
\end{aligned}
$$

where $\left(S_{i j}\right)_{0}$ and $(A)_{0}$ are a particular solution to the equation, while $\lambda \in \mathbb{Z}$.

It is important to note that the shifting parameters $S_{i j}$ are constrained as seen in Definition 7, and thus, $S_{i j} \in\left\{0, \ldots, \operatorname{Sym}\left(\mathcal{G}_{i}\right)-1\right\}$. This means that the number of different $S_{i j}$ available is:

$$
\begin{aligned}
\left\lfloor\frac{\operatorname{Sym}\left(\mathcal{G}_{i}\right)-1}{\Delta l}\right\rfloor+1 & =\left\lfloor\frac{\left(\operatorname{Sym}\left(\mathcal{G}_{i}\right)-1\right) \operatorname{gcd}\left(\operatorname{Sym}\left(\mathcal{G}_{i}\right), L_{j j}\right)}{\operatorname{Sym}\left(\mathcal{G}_{i}\right)}\right\rfloor+1 \\
& =\operatorname{gcd}\left(\operatorname{Sym}\left(\mathcal{G}_{i}\right), L_{j j}\right)-\left\lceil\frac{\operatorname{gcd}\left(\operatorname{Sym}\left(\mathcal{G}_{M}\right), L_{\Omega}\right)}{\operatorname{Sym}\left(\mathcal{G}_{M}\right)}\right\rceil+1 \\
& =\operatorname{gcd}\left(\operatorname{Sym}\left(\mathcal{G}_{i}\right), L_{j j}\right) .
\end{aligned}
$$

The condition provided by Eq. (102) as well as the number of different values of $S_{i j}$ given by Eq. (103) must be ful- filled for all the dimensions of the space, thus, the condition for the existence of solution is:

$$
\operatorname{gcd}\left(\operatorname{Sym}\left(\mathcal{G}_{i}\right), L_{j j}\right) \mid\left(L_{i j}-\sum_{q=j+1}^{i-1} S_{i j} L_{q j}\right), \forall\{i, j: i>j\},
$$

while the total number of different configurations is given by all possible combinations of the shifting parameters $S_{i j}$. Therefore the number of different configurations is:

$\prod_{i=2}^{n} \prod_{j=1}^{i-1} \operatorname{gcd}\left(\operatorname{Sym}\left(\mathcal{G}_{i}\right), L_{j j}\right)$.

As it can be observed, if all the necklaces comprise all the available positions of their respective dimensions, we obtain that there exists congruent configurations always since $\operatorname{Sym}\left(\mathcal{G}_{i}\right)=1$ for any dimension. In addition, there is only one possible configuration due to the fact that:

$$
\prod_{i=2}^{n} \prod_{j=1}^{i-1} \operatorname{gcd}\left(1, L_{j j}\right)=1
$$

This is an expected result, since in a complete configuration, a given Hermite Normal Form can only generate one congruent lattice distribution.

\subsubsection{Fixing $\mathcal{G}_{i}$ and $L_{i i}$ for all $i \in\{1, \ldots, n\}$}

In this second case of study, we are interested in determine, for a space of fixed size, the number of different distributions that can be generated using a given set of patterns. In order to impose that condition, the number of available positions in each dimension, that is, the parameters $L_{i i}$ from the Hermite Normal Form, and the necklaces $\mathcal{G}_{i}$ are fixed. Note that this condition is equivalent to determine the number of pairs $\left\{S_{i j}, L_{i j}\right\}$ that fulfill Eq. (63).

Theorem 9. Given a set of necklaces $\mathcal{G}_{i}$ and a number of available positions in each dimension $L_{i i}$, the number of different congruent configurations that can be generated is:

$\prod_{i=1}^{n} L_{i i}^{(n-i)}$

Proof. We first focus on a particular combination of dimension of study $i$ and dimension in which rotations are considered. Then, by using Eq. (63) we can present the relation between $S_{i j}$ and $L_{i j}$ :

$L_{j j} S_{i j}-1 L_{i j}=\operatorname{ASym}\left(\mathcal{G}_{M}\right)-\sum_{q=j+1}^{i-1} S_{i q} L_{q j}$,

where the elements $S_{i q}$ and $L_{q j}$ with $q>j$ are already known since they were computed in previous recursions of the equation (that is, in the equations related to previous dimensions). Eq. (108) can be regarded as a Diophantine equation where $S_{i j}$ and $L_{i j}$ are its variables, and whose solution exists if and only if: 
$\operatorname{gcd}\left(L_{j j}, 1\right) \mid A \operatorname{Sym}\left(\mathcal{G}_{M}\right)-\sum_{q=j+1}^{i-1} S_{i q} L_{q j}$

which is always true. Furthermore, the solutions generated are:

$$
\begin{aligned}
& \left(S_{i j}\right)_{\lambda}=\left(S_{i j}\right)_{0}+\lambda, \\
& \left(L_{i j}\right)_{\lambda}=\left(L_{i j}\right)_{0}+\lambda L_{j j},
\end{aligned}
$$

where $\left(S_{i j}\right)_{0}$ and $\left(L_{i j}\right)_{0}$ are a particular solution of Eq. (108) and $\lambda \in \mathbb{Z}$. By using Eq. (110), it can be concluded that, given a value of $A$, there only exists one solution to the equation. This is due to the fact that $L_{i j} \in\left\{0, \ldots, L_{j j}-1\right\}$ and that the summation is assumed to be fixed in the equation. Therefore, we now focus on the determination of all $A$ values that are compatible with the conditions.

From Eq. (108) and the different maximum and minimum constraints of $L_{i j}$ and $S_{i j}$ (see Theorem 3 and Definition 7), the maximum and minimum values of $A$ that can be obtained are:

$$
\begin{aligned}
& A_{\text {min }}=\frac{1}{\operatorname{Sym}\left(\mathcal{G}_{i}\right)}\left(\sum_{q=j+1}^{i-1} S_{i q} L_{q j}-\left(L_{j j}-1\right)\right), \\
& A_{\max }=\frac{1}{\operatorname{Sym}\left(\mathcal{G}_{i}\right)}\left(\sum_{q=j+1}^{i-1} S_{i q} L_{q j}+L_{j j}\left(\operatorname{Sym}\left(\mathcal{G}_{i}\right)-1\right)\right),
\end{aligned}
$$

which define:

$\left\lfloor A_{\max }-A_{\text {min }}\right\rfloor+1=\left\lfloor\frac{L_{j j}\left(\operatorname{Sym}\left(\mathcal{G}_{i}\right)-1\right)+\left(L_{j j}-1\right)}{\operatorname{Sym}\left(\mathcal{G}_{i}\right)}\right\rfloor+1=L_{j j}$

different possible values of $A$. This means that, there are $L_{j j}$ different combinations for the pairs $\left\{L_{i j}, S_{i j}\right\}$. It is interesting to note that this is coincident with the number of possibilities that $L_{i j} \in\left\{0, \ldots, L_{j j}-1\right\}$ presents.

Therefore, and under these conditions, the formulation is able to generate a number of different configurations equal to:

$\prod_{i=1}^{n} L_{i i}^{(n-i)}$,

which corresponds to the number of possible configurations of a complete congruent lattice (see Theorem 4).

Theorem 9 also leads to an important property when both the set of necklaces $\mathcal{G}_{i}$ and the number of available positions in each dimension $L_{i i}$ is fixed: the number of possibilities only depends on the number of available positions, not in the necklace selected nor in the configuration numbers.

\subsubsection{Fixing $N_{i}$ and $L_{i i}$ for all $i \in\{1, \ldots, n\}$}

The problem studied here has some similarities with previous counting methodology, however, instead of fixing the necklaces $\mathcal{G}_{i}$ (as done previously), the number of elements taken in each dimension is set, that is, $N_{i}=\left|\mathcal{G}_{i}\right|$. Therefore, this counting process provides the number of distribution possibilities that are available with a given number of elements retrieved and a set of available positions in each dimension.

Theorem 10. Let $L_{i i}$ where $i \in\{1, \ldots, n\}$ be the number of available positions in each dimension $i$ of a n-dimensional space, and let $N_{i}$ be number of elements taken as a subset of the available positions. Then, the number of different congruent lattice configurations using necklaces that can be generated is:

$$
\prod_{i=1}^{n} L_{i i}^{(n-i)} \sum_{\substack{g=1 \\
g_{i i} \\
\frac{L_{i i}}{g} N_{i}}}^{L_{i i}} \frac{1}{g}\left[\left(\begin{array}{c}
g \\
\frac{N_{i}}{L_{i i}} g
\end{array}\right)-\sum_{\substack{g^{\prime}=1 \\
g^{\prime}\left|g \\
L_{i i j}\right| N_{i} \\
g^{\prime} \mid N_{i}}}^{g-1} \frac{g^{\prime}}{L_{i i}}\left|\operatorname{Fix}\left(g^{\prime}\right)\right|\right],
$$

where for each dimension $i$, the term $|\operatorname{Fix}(g)|$ represents the number of elements contained in the Fix of $g$, which can be obtained using:

$$
|\operatorname{Fix}(g)|=\frac{L}{g}\left[\left(\begin{array}{c}
g \\
\frac{N}{L} g
\end{array}\right)-\sum_{\substack{g^{\prime}=1 \\
g^{\prime} \mid g \\
\frac{L}{g^{\prime} N}}}^{g-1} \frac{g^{\prime}}{L}\left|\operatorname{Fix}\left(g^{\prime}\right)\right|\right] .
$$

Proof. From Theorem 7 we know that in each dimension $i$, it is possible to generate:

$$
\mathcal{P}_{i}=\sum_{\substack{g=1 \\
g\left|L_{i i} \\
\frac{L_{i i} \mid N_{i}}{g}\right| N_{i}}}^{L_{i i}} \frac{1}{g}\left[\left(\begin{array}{c}
g \\
\frac{N_{i}}{L_{i i}} g
\end{array}\right)-\sum_{\substack{g^{\prime}=1 \\
g^{\prime}\left|g \\
\frac{L_{i j} \mid}{g^{\prime}}\right| N_{i}}}^{g-1} \frac{g^{\prime}}{L_{i i}}\left|\operatorname{Fix}\left(g^{\prime}\right)\right|\right],
$$

different necklaces, where:

$$
|\operatorname{Fix}(g)|=\frac{L_{i i}}{g}\left[\left(\begin{array}{c}
g \\
\frac{N_{i}}{L_{i i}} g
\end{array}\right)-\sum_{\substack{g^{\prime}=1 \\
g^{\prime} \mid g \\
\frac{L_{i j} \mid N_{i}}{g^{\prime}}}}^{g-1} \frac{g^{\prime}}{L_{i i}}\left|\operatorname{Fix}\left(g^{\prime}\right)\right|\right] \text {. }
$$

This means that the total number of combinations of necklaces between the different dimensions is:

$\prod_{i=1}^{n} \mathcal{P}_{i}$

In addition, once all the necklaces in the space are fixed, we can apply Theorem 9 in order to compute the number of different configurations by a direct combination of all the possibilities: 
$\prod_{i=1}^{n} \mathcal{P}_{i} \prod_{j=1}^{n} L_{j j}^{(n-j)}$

The former expression can be rearranged into:

$\prod_{i=1}^{n} \mathcal{P}_{i} L_{i i}^{(n-i)}$

and using Eq. (116) we obtain:

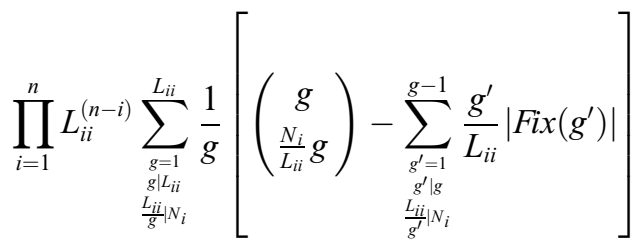

which shows the total number of configurations that are presented in the lattice under the considered conditions of fixed $N_{i}$ and $L_{i i}$.

In addition, it is interesting to note that if $N_{i}=L_{i i}$ for all $i$, the number of configurations is the same as in the case of a complete lattice (as seen in Theorem 4). This result was expected due to the fact that the necklace methodology is a generalization of the original lattice methodology presented.

\section{Alternative formulation}

It is possible to generate a congruent lattice, and its generalization using necklaces, based on an entirely integer formulation. This includes the variables $V_{i}$ which are real numbers in the original formulation.

We depart from Eq. (14):

$\sum_{j=1}^{i} L_{i j} V_{j}=k_{i}$

and multiply each expression by the integer $\prod_{p=1}^{i-1} L_{p p}$, obtaining:

$\sum_{j=1}^{i} L_{i j} \prod_{p=1}^{i-1} L_{p p} V_{j}=k_{i} \prod_{p=1}^{i-1} L_{p p}$

which can be expressed in a different manner by simple operations in the summation:

$L_{i i} \prod_{p=1}^{i-1} L_{p p} V_{i}+\sum_{j=1}^{i-1} L_{i j} \prod_{p=1}^{i-1} L_{p p} V_{j}=k_{i} \prod_{p=1}^{i-1} L_{p p}$

and by merging the product in the first term of the equation and rearranging the product of the second term we derive:

$\prod_{p=1}^{i} L_{p p} V_{i}+\sum_{j=1}^{i-1} L_{i j} \prod_{m=j+1}^{i-1} L_{m m} \prod_{p=1}^{j} L_{p p} V_{j}=k_{i} \prod_{p=1}^{i-1} L_{p p}$.

Now, a change in the distribution variable $V_{i}$ is performed. Let $\mathcal{N}_{i}$ be the new set of variables that relate with the originals $V_{i}$ through the following relation:
$\mathcal{N}_{i}=\prod_{p=1}^{i} L_{p p} V_{i}$

which introduced in Eq. (125) leads to:

$\mathcal{N}_{i}+\sum_{j=1}^{i-1} L_{i j} N_{j} \prod_{m=j+1}^{i-1} L_{m m}=k_{i} \prod_{p=1}^{i-1} L_{p p}$

where we can obtain the value of the new variable by means of the distribution parameters $k_{i}$ :

$\mathcal{N}_{i}=k_{i} \prod_{p=1}^{i-1} L_{p p}-\sum_{j=1}^{i-1} L_{i j} N_{j} \prod_{m=j+1}^{i-1} L_{m m}$.

As in the case of $V_{i}$, the former expression is constrained due to the modular arithmetic of the space. In particular, and since $\mathcal{N}_{i}=\prod_{p=1}^{i} L_{p p} V_{i}, \mathcal{N}_{i} \in\left[1, \prod_{p=1}^{i} L_{p p}\right]$ :

$\mathcal{N}_{i}=\bmod \left[k_{i} \prod_{p=1}^{i-1} L_{p p}-\sum_{j=1}^{i-1} L_{i j} N_{j} \prod_{m=j+1}^{i-1} L_{m m}, \prod_{p=1}^{i} L_{p p}\right]$.

One important thing to note is that $\mathcal{N}_{i} \in \mathbb{N} \forall i \in \mathbb{Z}_{n}$, since all the operations performed are products and sums of integer numbers.

Now, we introduce the new formulation provided by Eq. (129) into the necklace formulation given by Eq. (57), which leads to:

$$
\begin{aligned}
& \mathcal{N}_{i}+\sum_{j=1}^{i-1} L_{i j} N_{j} \prod_{m=j+1}^{i-1} L_{m m}=k_{i} \prod_{p=1}^{i-1} L_{p p} \\
& k_{i}=\bmod \left[\mathcal{G}_{i}\left(k_{i}^{*}\right)+\bmod \left(\sum_{j=1}^{i-1} S_{i j} k_{j}, \operatorname{Sym}\left(\mathcal{G}_{i}\right)\right), L_{i i}\right],
\end{aligned}
$$

where the congruent configurations correspond to the ones provided by Theorem 6 . In that respect, note that the congruent relations provided by Eq. (63) are independent on the definition of the variables used.

Eq. (130) defines congruent lattices where the variables $\mathcal{N}_{i}$ present now integer numbers (note that they are sums and products of integers) that are defined in $\mathcal{N}_{i} \in\left[1, \ldots, \prod_{p=1}^{i} L_{p p}\right]$.

\section{Relation with Lattice and Necklace Flower Constellations}

$n$-Dimensional congruent lattices using necklaces is the mathematical generalization of the 2D, 3D and 4D Lattice and Necklace Flower Constellations. In particular, the ndimensional congruent distribution (see Section 3) presents the general formulation for the Lattice Flower Constellations, providing also the constraints of the distribution parameters and the number of possibilities that Lattice Flower Constellations are able to generate. On the other hand, $n$-Dimensional congruent lattices using necklaces (see Section 4) does the same for the Necklace Flower Con- 
stellations. It is also important to note that Lattice Flower Constellations are also a generalization of other well known satellite constellation designs, including Walker Constellations or Draim Constellations, and thus, the $n$ dimensional congruent lattices using necklaces is also a generalization of these methodologies, being the theorems presented in this work also of application to these constellation design techniques.

2D and 3D Lattice and Necklace Flower Constellations perform their satellite distribution using just angles as distribution variables: the right ascension of the ascending node, the argument of perigee and the mean anomaly; being the other design variables (semi-major axis, eccentricity and inclination) common for all the satellites of the constellation. This means that all the dimensions of the problem where the distribution is performed have modular arithmetic $\bmod (2 \pi)$ (they are angles), and thus, the configuration space becomes a torus where satellites are distributed. It is in this space where the distribution is performed by searching for congruent and uniform distributions (which are the ones with better properties for global coverage (Casanova et al., 2014a)).

On the other hand, 4D Lattice and Necklace Flower Constellations (Arnas, 2018) are defined using the alternative formulation presented in Section 5. This is due to the fact that the semi-major axis of the orbits (the new distribution variable in the 4D Lattice and Necklace Flower Constellations) does not have modular arithmetic. Nevertheless, the formulation allows to define different altitudes for the constellation in such a way that some properties of periodicity and uniformity are imposed. This shows an example of the multiple possibilities of application of the alternative formulation presented in this work.

Moreover, it is worth noting that $n$-dimensional congruent lattices using necklaces allows to increase the number of possibilities in Lattice and Necklace Flower Constellations. This is due to two generalizations introduced in this methodology. First, $n$-dimensional congruent lattices using necklaces allows to freely select the hierarchy order of the distribution variables, that is, the order of dependency between dimensions. Second, the methodology also allows to set the values that the distribution variables can present, providing more freedom to the designer. In addition, and based on the counting theorems presented in this work, the methodology allows to provide direct control in the number of possible configurations that will be generated, a property extremely useful in optimization problems since the size of the searching space is known beforehand.

\subsection{Example of application to the $2 D$ formulation of Flower Constellations}

In order to exemplify this relation, we show a summary of its application to 2D Lattice Flower Constellations. By using Theorem 1 for the case of two dimensions and a space modulo $2 \pi$ :

$$
\begin{aligned}
\Delta \Omega_{i j} & =2 \pi V_{1}=2 \pi \frac{i}{L_{11}} \bmod (2 \pi), \\
\Delta M_{i j} & =2 \pi V_{2}=2 \pi\left(\frac{j}{L_{22}}-\frac{L_{21}}{L_{22}} \frac{i}{L_{11}}\right) \bmod (2 \pi),
\end{aligned}
$$

where $k_{1}=i$ and $k_{2}=j$ names a satellite in orbit $i$ and position $j$ in that orbit. In here, the first dimension represents the distribution in the right ascension of the ascending node of the satellites (which determines the orbital plane in which spacecrafts are located), while the second dimension is the mean anomaly (which determines the position of the satellites in each orbital plane). That way, $L_{11}$ defines the number of equally spaced orbital planes in which the constellation is distributed, while $L_{22}$ is the number of satellites that are uniformly distributed in each orbit. Finally, the only combination number that appears in this formulation $L_{21}$ determines the relative spacing between spacecrafts at adjacent orbital planes. As it can be seen, this formulation is equivalent to the ones presented in Avendaño et al. (2013) for the 2D Lattice Flower Constellations formulation, or to a Walker-Delta constellation (Walker, 1984) for the case of circular orbits.

As a simple example of application of this formulation, a constellation in 3 orbital planes with 5 satellites per orbit ( $L_{11}=3$ and $L_{22}=5$ respectively) is defined. As we know from Theorem 4, the number of different possibilities of design that a distribution in mean anomaly and right ascension of the ascending node can provide is $L_{11}=3$, which correspond to the three possible values of the combination number $L_{21}=\{0,1,2\}$. The selection of $L_{21}$ determines the relative phasing of satellites between orbital planes, that is, the phasing between the mean anomaly (dimension 2) with respect to the right ascension of the ascending node (dimension 1).

Figs. 2-4 shows the three possible combinations of constellations for these conditions. Particularly, Fig. 2 represents the case with $L_{21}=0$, Fig. 3 the case $L_{21}=1$, and Fig. 4 the case $L_{21}=2$. These three figures contain both the inertial distribution of the constellation for a given instant and also the $(\Omega, M)$-space representation of the distribution. As it can be seen, all distributions are uniform and congruent. This implies also, that each satellite from the constellation observes exactly the same relative distribution, that is, if we select any satellite of the constellation as the reference satellite for the distribution, the $(\Omega, M)$ space representation of the constellation is exactly the same. Note also that these three configurations presented are the only ones that fulfill all the considered conditions of uniformity and congruence.

If instead, the necklace formulation is considered, $L_{11}$ and $L_{22}$ become the parameters that determine now the number of available orbital planes and positions in each orbit, while $N_{1}$ and $N_{2}$ define the actual number of orbital planes and positions in each orbit in use. Finally, $L_{21}$ and $S_{21}$ are the parameters that control the relative phasing of satellites between adjacent planes. In particular, applying Theorem 5 to a two dimensional space modulo $2 \pi$ : 

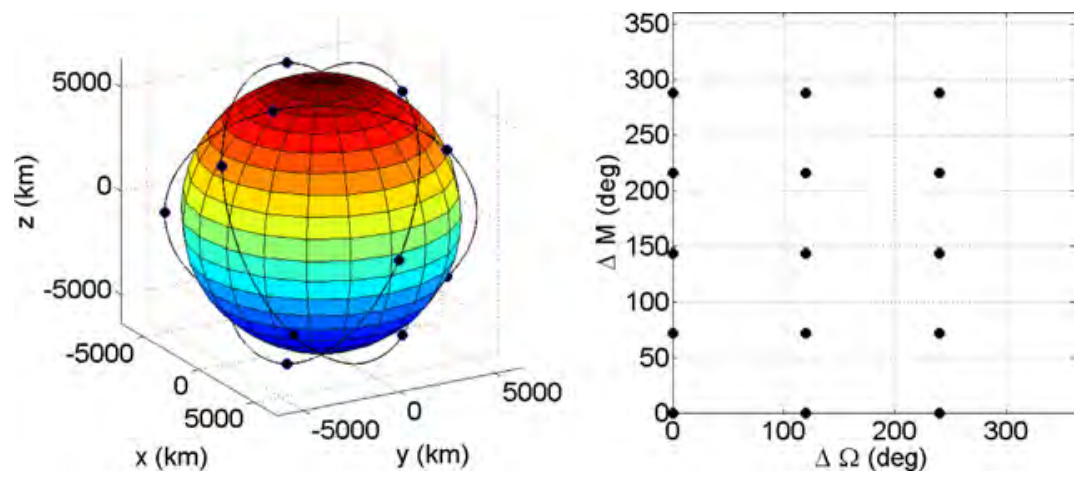

Fig. 2. Constellation distribution, case $L_{21}=0$.
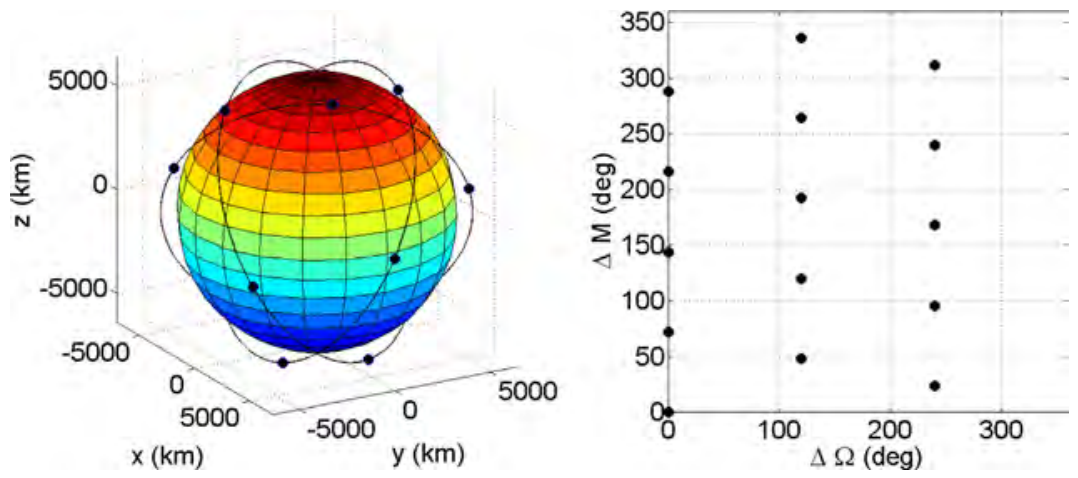

Fig. 3. Constellation distribution, case $L_{21}=1$.
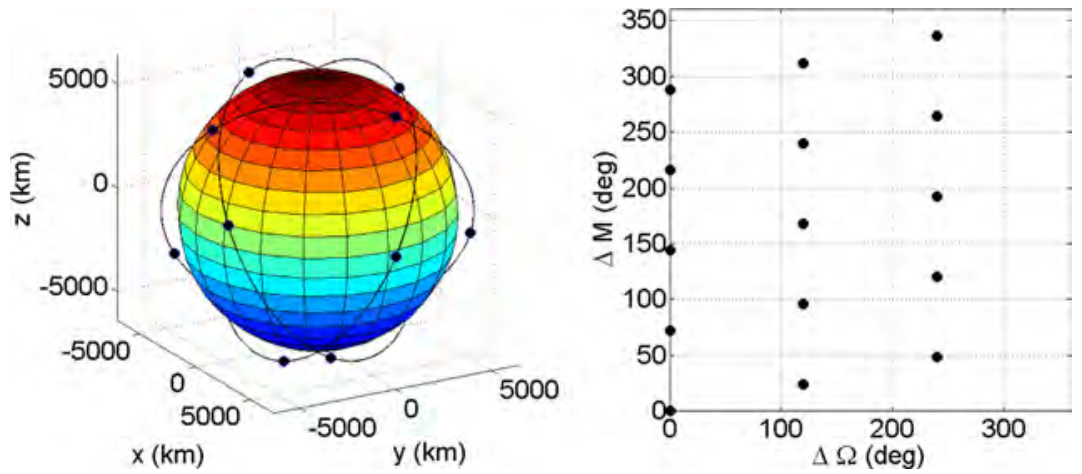

Fig. 4. Constellation distribution, case $L_{21}=2$.

$\begin{aligned} L_{11} V_{1} & =\mathcal{G}_{\Omega}\left(i^{*}\right) \\ L_{21} V_{1}+L_{22} V_{2} & =\bmod \left[\mathcal{G}_{M}\left(j^{*}\right)+S_{21} \mathcal{G}_{\Omega}\left(i^{*}\right), L_{22}\right]\end{aligned}$

which after some algebraic manipulation, leads to the following equation:

$\Delta \Omega_{i^{*} j^{*}}=2 \pi V_{1}=2 \pi \frac{\mathcal{G}_{\Omega}\left(i^{*}\right)}{L_{11}} \bmod (2 \pi) ;$

$$
\begin{aligned}
\Delta M_{i^{*} j^{*}} & =2 \pi V_{2} \\
& =\frac{2 \pi}{L_{22}}\left[\mathcal{G}_{M}\left(j^{*}\right)+S_{21} \mathcal{G}_{\Omega}\left(i^{*}\right)-L_{21} \frac{\mathcal{G}_{\Omega}\left(i^{*}\right)}{L_{11}}\right] \bmod (2 \pi),
\end{aligned}
$$

which is an equivalent expression to the one found in Arnas et al. (2017a) for the case of 2D Necklace Flower Constellations with necklaces both in the mean anomaly $\left(\mathcal{G}_{M}\right)$ and in the right ascension of the ascending node $\left(\mathcal{G}_{\Omega}\right)$.

Following the previous example, we are still looking for constellations distributed in 3 different orbital planes $\left(N_{11}=3\right)$ and 5 satellites per orbit $\left(N_{22}=5\right)$. However, this time we are interested in expanding the number of possibilities of design by allowing some non-uniformities in the configuration while still maintaining the congruent property. That way, we have to select the size of the fictitious constellation, that is, the number of available positions in which satellites can be located. These available positions can be the result of the application of a set of mission requirements, or a given slot strategy defined for the con- 


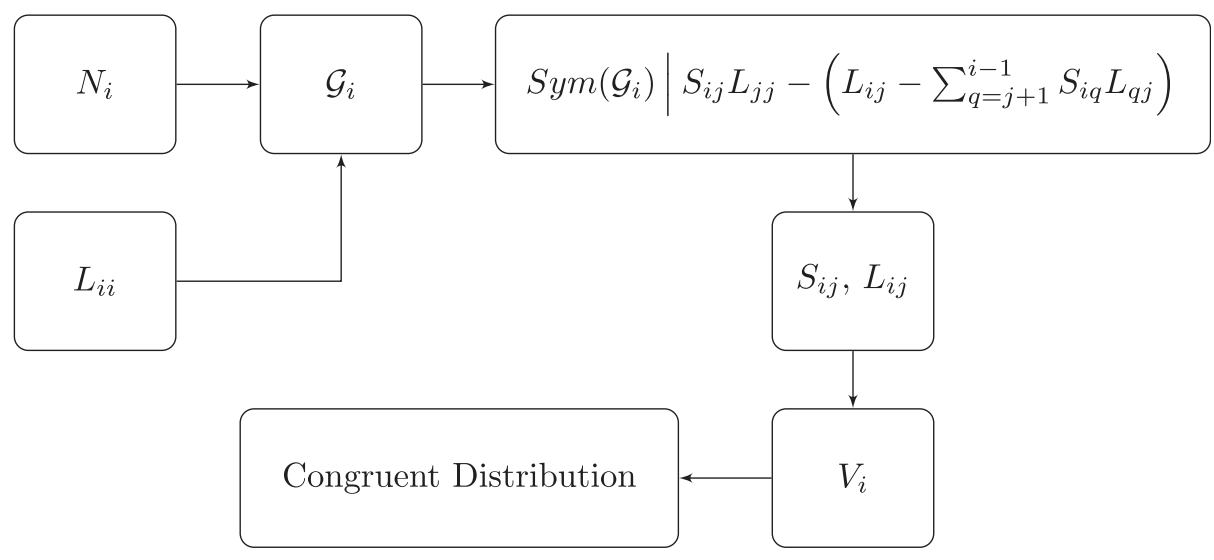

Fig. 5. Flowchart of the process for the generation of all configurations.

stellations. Suppose that we define a constellation with double the number of available positions in each dimension. This leads to $L_{11}=6$ and $L_{22}=10$. Under these conditions, using Theorem 10 we obtain that the number of different combinations is 4824 . If instead we are interested in a particular combination of necklaces, for instance $\mathcal{G}_{\Omega}=\{1,3,5\}$ and $\mathcal{G}_{M}=\{1,2,3,4,5\}$, then, we can use Theorem 9 to obtain that the number of possible configurations is reduced to $L_{11}=6$. This situation can appear when we are interested in a given revisiting time or a general structure for the constellation. Finally, if we require a precise phasing between orbital planes, for instance to impose that all satellites share the same ground-track (Arnas and Casanova, 2020), then we have to fix $L_{21}$ and thus, the Hermite Normal Form is completely defined. Therefore, by using Theorem 8 we can obtain that the number of possible configurations is $\operatorname{gcd}\left(\operatorname{Sym}\left(\mathcal{G}_{M}\right), L_{11}\right)=2$ if the value of $L_{21}$ is either 0,2 or 4. As it can be seen, the number of possible constellations is significantly increased with respect to the lattice formulation. Note also that we can increase as much as we want the number of available positions, and therefore, the number of different distribution possibilities that the formulation provides.

\section{Generation of all the configurations}

This section contains a general overview of the process required to generate all the possible configurations that the $n$-Dimensional congruent lattices using necklaces is able to provide. The flowchart provided in Fig. 5 summarizes the process.

The first step in the generation process is to fix the number of different elements that will appear in each dimension of the problem $\left(N_{i}\right)$. This will set the number of elements that are distributed during the process (remember that the total number of elements in the distribution is $\left.\prod_{i=1}^{n} N_{i}\right)$. Then, the number of available positions is each dimension is defined $\left(L_{i i}\right)$, which determines the size of the searching space and also the number of different possibilities that the methodology will generate. From both sets of quantities $\left(N_{i}\right.$ and $\left.L_{i i}\right)$ we generate all possible necklaces $\left(\mathcal{G}_{i}\right)$ for each dimension using a generation algorithm (Cattell et al., 2000; Sawada, 2003). Then, Theorem 6 is applied on each dimension, taking into account that the system of equations provided by this theorem can be easily solved sequentially. That way, all the compatible values of shifting parameters $S_{i j}$ and configuration numbers $L_{i j}$ that allow to generate all the congruent distributions are obtained. Once this generation process is performed, we only require to select one of the possible configurations and compute the location in each dimension of every element of the distribution $\left(V_{i}\right)$ using Theorem 5.

\section{Conclusions}

This work introduces the $n$-dimensional congruent lattices using necklaces, a complete generalization to $n$ dimensions of the mathematical basis behind the 2D, 3D and 4D Lattice and Necklace Flower Constellations, Walker Constellations and Draim Constellations. It also represents the mathematical foundation of that set of satellite constellation design techniques.

In its general form, this methodology is able to generate all the congruent and uniform distributions in a $n$ dimensional space subjected to modular arithmetic. Moreover, this methodology allows to select the subset of elements that are able to maintain the properties of uniformity, symmetry and congruence in the resultant configuration. In addition, and for the cases where we do not have a natural modular arithmetic in the problem, but instead a given set of available positions, the methodology is able to perform congruent distributions of elements over these available positions using a complete integer formulation.

This manuscript introduces three sets of theorems. The first set provides the formulation to generate lattice and necklace congruent distributions in the space considered. The second set aims to determine the constraints that the distribution parameters must follow in order to avoid duplicates in the formulation. Finally, the third set consists of several counting theorems that focus on the evaluation 
of the number of different possibilities of design that this methodology is able to generate under the condition of congruence. In that sense, the most common cases of study are considered.

$n$-Dimensional congruent lattices using necklaces provides a deep mathematical foundation for the configurations presented in the Lattice and Necklace Flower Constellations theories, as well as in any other uniform congruent distribution in a modular $n$-dimensional space. Moreover, and compared with Lattice and Necklace Flower Constellations formulations, $n$-dimensional congruent lattices using necklaces allows to increase the freedom in design since it allows to change the order of the distribution variables and also to define a wider variety of configurations. In that respect, this work complements the methodology presented for the Necklace Flower Constellations, providing mathematical robustness to the design methodology and a more compact formulation for the Lattice and Necklace Flower Constellations.

Finally, note that the formulation and theorems presented in this manuscript have applications not only to satellite constellation design, but also to the fields of Combinatorics and Number Theory. In that sense, the results included in this work provide a complete set of tools and theorems to deal with the generation and study of uniform distributions and necklaces.

\section{Acknowledgments}

David Arnas, Daniel Casanova and Eva Tresaco acknowledge the support of the Spanish Ministry of Economy and Competitiveness, Project No. ESP2017-87113-R (AEI/FEDER, UE); and the Aragon Government and European Social Fund (group E24_17R).

\section{References}

Apostol, T.M., 1976. Introduction to Analytic Number Theory. SpringerVerlag, New York.

Arnas, D., 2018. Necklace Flower Constellations Ph.D. thesis. Universidad de Zaragoza.

Arnas, D., Casanova, D., 2020. Nominal definition of satellite constellations under the Earth gravitational potential. Celest. Mech. Dyn. Astron. 132, 19.

Arnas, D., Casanova, D., Tresaco, E., 2016. Relative and absolute stationkeeping for two-dimensional-lattice flower constellations. J. Guid. Control Dyn., 2602-2604

Arnas, D., Casanova, D., Tresaco, E., 2017a. 2D Necklace Flower Constellations. Acta Astronaut. 142, 18-28.

Arnas, D., Casanova, D., Tresaco, E., 2017b. Time distributions in satellite constellation design. Celest. Mech. Dyn. Astron. 128 (2-3), $197-219$.

Arnas, D., Casanova, D., Tresaco, E., Mortari, D., 2017c. 3-Dimensional Necklace Flower Constellations. Celest. Mech. Dyn. Astron. 129 (4), 433-448.

Arnas, D., Fialho, M.A., Mortari, D., 2017d. Fast and robust kernel generators for star trackers. Acta Astronaut. 134, 291-302.

Avendaño, M.E., Davis, J.J., Mortari, D., 2013. The 2-D lattice theory of Flower Constellations. Celest. Mech. Dyn. Astron. 116 (4), 325-337.
Avendaño, M.E., Mortari, D., 2012. New insights on flower constellations theory. IEEE Trans. Aerosp. Electron. Syst. 48 (2), 1018-1030.

Ballard, A.H., 1980. Rosette constellations of earth satellites. IEEE Trans. Aerosp. Electron. Syst. 5, 656-673.

Beste, D.C., 1978. Design of satellite constellations for optimal continuous coverage. IEEE Trans. Aerosp. Electron. Syst. 3, 466-473.

Birkhoff, G., 1935. On the structure of abstract algebras. Math. Proc. Cambridge Philos. Soc. 31 (4), 433-454.

Casanova, D., Avendaño, M., Mortari, D., 2014a. Seeking gdop-optimal flower constellations for global coverage problems through evolutionary algorithms. Aerosp. Sci. Technol. 39, 331-337.

Casanova, D., Avendaño, M.E., Mortari, D., 2014. Design of flower constellations using necklaces. IEEE Trans. Aerosp. Electron. Syst. 50 (2), 1347-1358.

Casanova, D., Avendaño, M., Tresaco, E., 2015. Lattice-preserving flower constellations under j2 perturbations. Celest. Mech. Dyn. Astron. 121 (1), 83-100.

Cattell, K., Ruskey, F., Sawada, J., Serra, M., Miers, C.R., 2000. Fast algorithms to generate necklaces, unlabeled necklaces, and irreducible polynomials over gf (2). J.Algorithms 37 (2), 267-282.

Conway, J., Sloane, N., 1999. Sphere Packings, Lattices and Groups, vol. 290. Springer-Verlag, New York.

Dale, P.C., Rinaldi, S.P., Storz, M.F., 2017. Global positioning system performance optimization using a normalized function on configuration classes. J. Guid. Control Dyn. 40 (5), 1166-1175.

Davis, J.J., Avendaño, M.E., Mortari, D., 2013. The 3-d lattice theory of flower constellations. Celest. Mech. Dyn. Astron. 116 (4), 339-356.

Draim, J.E., 1987. A common-period four-satellite continuous global coverage constellation. J. Guid. Control Dyn. 10 (5), 492-499.

Gilbert, E.N., Riordan, J., 1961. Symmetry types of periodic sequences, Illinois J. Math. 5 (4), 657-665.

Lo, M.W., 1999. Satellite-constellation design. Comput. Sci. Eng. 1 (1), $58-67$.

Luders, R.D., 1961. Satellite networks for continuous zonal coverage. ARS J. 31 (2), 179-184.

Mortari, D., De Sanctis, M., Lucente, M., 2011. Design of flower constellations for telecommunication services. Proc. IEEE 99 (11), 2008-2019.

Mortari, D., Wilkins, M., Bruccoleri, C., 2004. The Flower Constellations. J. Astronaut. Sci. 52, 107-127.

Mortari, D., Wilkins, M.P., 2008. Flower constellation set theory. Part i: Compatibility and phasing. IEEE Trans. Aerosp. Electron. Syst. 44 (3), 953-962.

Mozhaev, G., 1973. The problem of the continuous earth coverage and the kinematically regular satellite networks. i. Cosm. Res. 11, 833-840.

Newman, M., 1972. Integral Matrices, vol. 45. Academic Press, New York and London.

Ortore, E., Cinelli, M., Circi, C., 2017. A ground track-based approach to design satellite constellations. Aerosp. Sci. Technol. 69, 458-464.

Riordan, J., 1978. An Introduction to Combinatorial Analysis. Princeton University Press, Princeton.

Sawada, J., 2003. A fast algorithm to generate necklaces with fixed content. Theoret. Comput. Sci. 301 (1-3), 477-489.

Storjohann, A., Labahn, G., 1996. Asymptotically fast computation of hermite normal forms of integer matrices. In: proceedings of the 1996 international symposium on Symbolic and algebraic computation, Zurich, 1996, ACM Press, pp. 259-266.

Tucker, A., 1984. Applied Combinatorics, vol. 287. Wiley, New York.

Ulybyshev, Y., 2008. Satellite constellation design for complex coverage. J. Spacecraft Rock. 45 (4), 843-849.

Walker, J., 1984. Satellite Constellations. J. Br. Interplanet. Soc. 37, 559572.

Wilkins, M.P., Mortari, D., 2008. Flower constellation set theory part ii: secondary paths and equivalency. IEEE Trans. Aerosp. Electron. Syst. $44(3), 964-976$. 